\title{
Notions de base sur la combustion
}

\section{Basic smattering on combustion}

\author{
Roger Prud'Homme
}

${ }^{1}$ Directeur de Recherche Emérite - Institut Jean Le Rond D’Alembert - Université Pierre et Marie Curie/CNRS

RÉSUMÉ. La découverte du feu a contribué à l'émergence de l'humanité. C'est ensuite la maîtrise scientifique de cette même combustion qui a permis à l'homme de se propulser sur la lune ou plus modestement de faire fonctionner le moteur de votre voiture. Qui n'a pas été fasciné par un feu d'artifice, une simple bougie ou un feu de bois ? De nombreux phénomènes physiques et chimiques s'y produisent que cet article va vous permettre de découvrir. ${ }^{2}$

La combustion résulte d'un ensemble de réactions chimiques qui libère de l'énergie. Les différents corps chimiques qui interviennent sont essentiellement un oxydant, un réducteur et les produits de combustion. Ces corps sont en mouvement, diffusent les uns dans les autres et la chaleur produite par les réactions chimiques se propage. La combustion est d'abord abordée en milieu gazeux. On distingue des flammes de diffusion et des flammes de prémélange. On étudie d'abord le cas laminaire, puis l'action de la turbulence sur ces flammes. Sont présentés ensuite sommairement quelques cas hétérogènes tels que la combustion de plaques, de sprays et de poudres.

ABSTRACT. The discovery of fire has contributed to the emergence of humanity. It is then the scientific mastery of this same combustion that allowed the man to propel himself on the moon or more modestly to operate the engine of your car. Who was not fascinated by fireworks, a candle or a fire? Many physical and chemical phenomena occur there that this article will allow you to discover.

The combustion results from a set of chemical reactions that releases energy. The different chemical bodies involved are essentially an oxidant, a reducing agent and the products of combustion. These bodies are in motion, diffuse into each other and the heat produced by chemical reactions spreads. The combustion is first approached in a gaseous medium. There are diffusion flames and premix flames. We first study the laminar case, then the action of turbulence on these flames. Then, a few heterogeneous cases are presented, such as the combustion of plates, sprays and powders.

MOTS-CLÉS. combustion, phase gazeuse, flamme de diffusion, flamme prémélangée, sprays, fusées à poudre.

KEYWORDS. combustion, gaseous phase, diffusion flame, premixed flames, sprays, solid rocket motors.

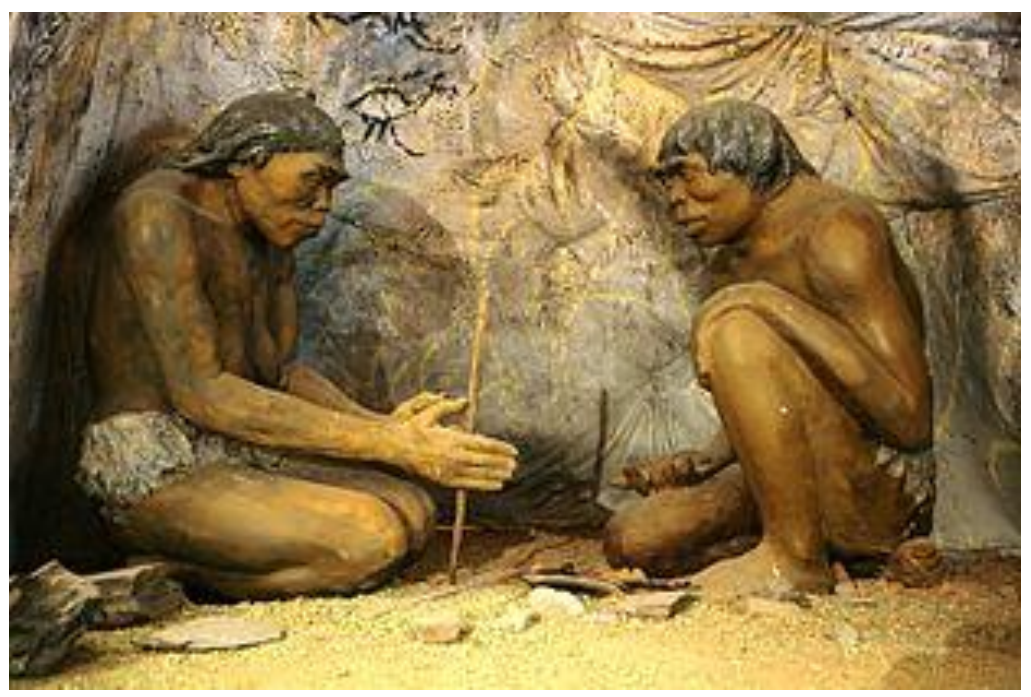

Production du feu par la technique de friction par rotation d'un foret (ou drille) sur une planchette de bois (diorama du musée national de Mongolie, https://fr.wikipedia.org/wiki/Domestication_du_feu)

${ }^{1}$ Sorbonne Université, CNRS, Institut Jean Le Rond d'Alembert, UMR 7190, F-75005 Paris, France - e-mail :

roger.prud homme@upmc.fr

2 L'article fait suite à une demande de R. Moreau et J. Sommeria en vue d'une utilisation pour l'Encyclopédie de

l'environnement https://www.encyclopedie-environnement.org/

(c) 2019 ISTE OpenScience - Published by ISTE Ltd. London, UK - openscience.fr 


\section{Le phénomène chimique}

La découverte du feu dans la préhistoire a créé une véritable révolution. L’énergie libérée par la combustion fournit de la chaleur permettant la cuisson des aliments, l'extraction et le travail des métaux - étapes importantes du développement humain - et mène aussi à l'émission de lumière, au début au moyen de torches, puis de bougies. Aujourd'hui la combustion a de nombreuses applications jusqu'au moteurs des lanceurs de satellites qui fonctionnent tous à l'énergie chimique. A ce propos, la propulsion par combustion est actuellement la seule disponible dans la phase de lancement et les motoristes cherchent à la rendre la plus efficace et la moins polluante possible. Notons que les moteurs cryotechniques (c'est-à-dire comprenant une étape de stockage en réservoir à très basse température) de la fusée Ariane fonctionnent avec de l'oxygène et de l'hydrogène dont le produit de combustion n'est pas polluant puisque c'est de l'eau.

La combustion résulte de réactions chimiques globalement exothermiques, c'est-à-dire qu'elles dégagent de la chaleur. Un exemple classique est la combustion du mélange $\mathrm{H}_{2}-\mathrm{O}_{2}$ qui produit de l'eau $\mathrm{H}_{2} \mathrm{O}$ avec un fort dégagement de chaleur. Cette réaction est en fait une chaîne de réactions qui fait intervenir des espèces chimiques intermédiaires fugitives comme le radical ${ }^{3} \mathrm{OH}^{\bullet}$ et les atomes isolés $\mathrm{H}$ et $\mathrm{O}$. Chacune des réactions de la chaîne ne dégage pas nécessairement de la chaleur, mais l'ensemble de la chaîne est exothermique.

a)

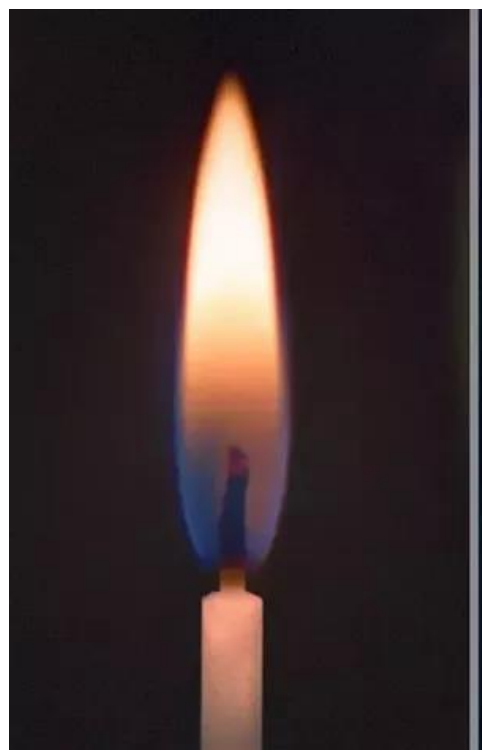

b)

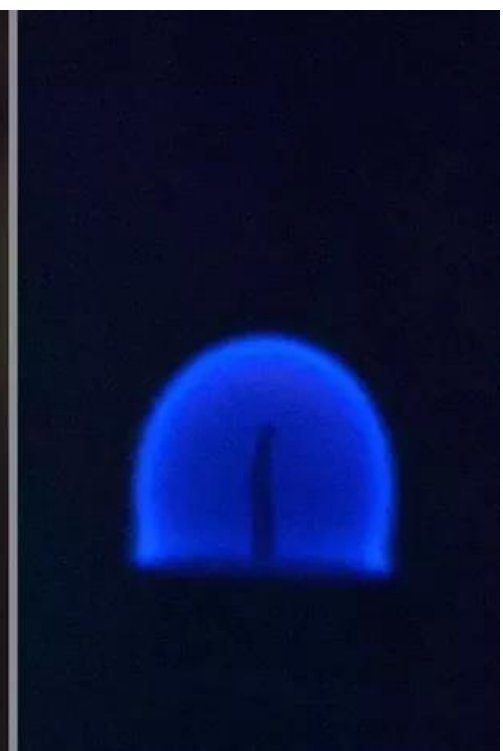

Figure 1. Comparaison d'une flamme a) sous pesanteur terrestre et b) en apesanteur dans l'ISS, https://commons.wikimedia.org/wiki/File:Candlespace.jpg

On pourra symboliser une réaction chimique simplement par $\mathrm{A} \rightarrow \mathrm{B}$ signifiant que les réactants $\mathrm{A}$ donnent les produits de réaction $\mathrm{B}$. Ou alors par $\mathrm{F}+\mathrm{O} \rightarrow \mathrm{P}$ où $\mathrm{F}$ est le fuel ou combustible, $\mathrm{O}$ est l'oxydant ou comburant, et $\mathrm{P}$ résume les produits de combustion. Mais une description plus détaillée est souvent nécessaire si l'on veut effectuer des calculs de cinétique chimique, c'est-à-dire déterminer les vitesses de réaction.

La vitesse d'une réaction est égale à une vitesse spécifique $k$ multipliée par les produits des concentrations des réactants élevées à la puissance de leur coefficient stœchiométrique. La vitesse spécifique de la réaction est une fonction de la température qui se met généralement sous la forme $k=\mathrm{B}$

\footnotetext{
${ }^{3}$ Un radical (souvent appelé radical libre) est une espèce chimique possédant un ou plusieurs électrons non appariés sur sa couche externe, ce qui le rend très réactif. Il se note par un point. 
$\mathrm{T}^{\mathrm{s}} \exp \left(-\mathrm{E}_{\mathrm{a}} / \mathrm{RT}\right)$, où $\mathrm{T}$ est la température absolue, $\mathrm{B}$ et $\mathrm{s}$ sont des constantes, $\mathrm{R}$ est la constante universelle des gaz parfaits $\left(\mathrm{R}=8,314 \mathrm{~J} \mathrm{~mole}^{-1} \mathrm{~K}^{-1}\right)$ et $\mathrm{E}_{\mathrm{a}}$ l'énergie d'activation de la réaction.

Ces vitesses dépendent fortement de la température car il faut apporter aux molécules une énergie suffisante, dite énergie d'activation, pour qu'elles puissent ensuite se recombiner en produisant de la chaleur. Ainsi un mélange d'hydrogène et d'oxygène ne réagit pas à température ordinaire, car les chocs moléculaires sont en général trop faibles pour briser les molécules $\mathrm{H}_{2}$ et $\mathrm{O}_{2}$, ce qui est nécessaire pour initier la réaction. A partir d'une certaine température, la réaction devient suffisamment active pour chauffer le milieu, et elle s'emballe très rapidement suite à la dépendance exponentielle de la vitesse de réaction avec la température.

La combustion de l'hydrogène se produit dans un milieu entièrement gazeux : c'est ce qu'on appelle une combustion en phase homogène. La flamme d'une cuisinière à gaz ou d'un bec Bunsen est également une combustion en phase homogène. La combustion d'un solide au contact de l'air est au contraire une combustion en phase hétérogène. Les incendies relèvent en général de ce cas ${ }^{4}$. Notons que la notion d'homogénéité est toute relative car elle dépend de l'échelle d'observation. Ainsi des poudres compressées sont homogènes à une échelle nettement supérieure à la taille des grains dont elles sont constituées. En revanche elles apparaissent hétérogènes si on les observe à l'échelle des grains de ces poudres.

La combustion est un phénomène complexe. Prenons l'exemple de la flamme de bougie (figure 1a). Celle-ci associe la fusion de la cire ou de la paraffine, le transport du liquide résultant de cette fusion dans la mèche poreuse, son évaporation suivie de sa combustion avec l'oxygène de l'air ambiant. La vapeur combustible diffuse à l'intérieur de la flamme dans les produits de combustion. L'oxygène diffuse à l'extérieur dans le mélange de produits de combustion et d'air. Le résultat est ce que l'on appelle flamme non prémélangée (ou flamme de diffusion). Les gaz chauds ont tendance à s'élever sous l'effet de la pesanteur (la force d'Archimède) du fait de leur densité plus faible, c'est ce que l'on appelle la convection naturelle.

Il arrive que cette flamme oscille, cela provient du développement d'une instabilité de la surface de contact entre les gaz brûlés en mouvement et l'air extérieur. Cette instabilité, dite de KelvinHelmholtz, qui a lieu également dans le cas de la flamme de prémélange obtenue avec un bec Bunsen, disparaît complètement en apesanteur. Notons à ce propos que les expériences en micropesanteur sont très instructives en physique car elles font disparaitre la poussée d'Archimède, ce qui change parfois l'aspect du phénomène (voir aussi la figure 4).

D'autre part, la présence d'oxydes de carbone $\mathrm{CO}$, de vapeur d'eau $\mathrm{H}_{2} \mathrm{O}$ et de suies rend la flamme lumineuse.

Cette luminosité change quelque peu en apesanteur car la convection naturelle - qui tend à allonger la flamme - disparaît et la flamme prend une forme presqu'hémisphérique. D'autre part sa couleur vire au bleuâtre comme le montre la figure $1 \mathrm{~b}$ : il s'agit en fait de l'authentique flamme de diffusion ${ }^{5}$. La diffusion alimente la flamme en oxygène, mais les produits de la combustion tels le $\mathrm{CO}_{2}$ ne sont plus entrainés par convection, ils restent au voisinage de la flamme et tendent à bloquer l'accès de l'oxygène, de sorte que la vitesse de combustion est ralentie et la flamme est moins chaude, ce qui explique sa couleur et qu'elle soit moins lumineuse. D'ailleurs elle ne survit pas très longtemps dans ces conditions, faute de gazéification du combustible.

${ }^{4}$ https://www.encyclopedie-environnement.org/air/comprendre-prevenir-feux-de-vegetation/

5 http://www.planete-sciences.org/blogs/pla-sci/2014/02/22/le-mystre-de-la-flamme-dans-lespace/ 


\section{Combustion non prémélangée laminaire}

\subsection{La flamme de bougie}

La flamme de bougie fait donc partie d'un processus complexe comprenant une flamme de diffusion. La figure 2 montre dans le détail ce qui se passe.

Ce que l'on appelle flamme ici est la surface de contact entre le combustible gazeux et l'oxygène de l'air: l'intérieur de la flamme, qui est creuse, est constitué de combustible quasi-pur tandis que l'extérieur est riche en oxygène. C'est le processus de diffusion ${ }^{6}$ qui amène ces deux composants au contact dans la zone de réaction, qui possède donc une certaine épaisseur. Celle-ci dépend de la diffusivité des gaz, mais aussi de la sensibilité de la réaction à la température (caractérisée par l'énergie d'activation $E_{a}$ de la réaction chimique). Des transferts diffusifs efficaces et une énergie d'activation élevée tendent à amincir les flammes. Dans le cas de la bougie, l'épaisseur est de l'ordre du millimètre.

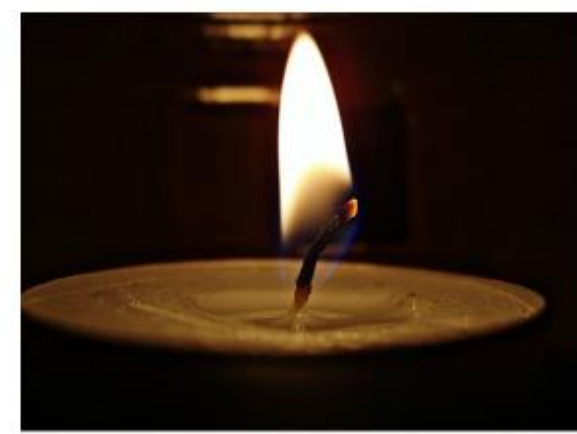

Diluant (azote) : N Comburant (oxygène) : $\mathrm{O}$ Produits de combustion: $\mathrm{P}$ Vapeur de combustible: $F_{G}$ Combustible liquide : $F_{L}$ Combustible solide : $\mathrm{F}_{\mathrm{S}}$

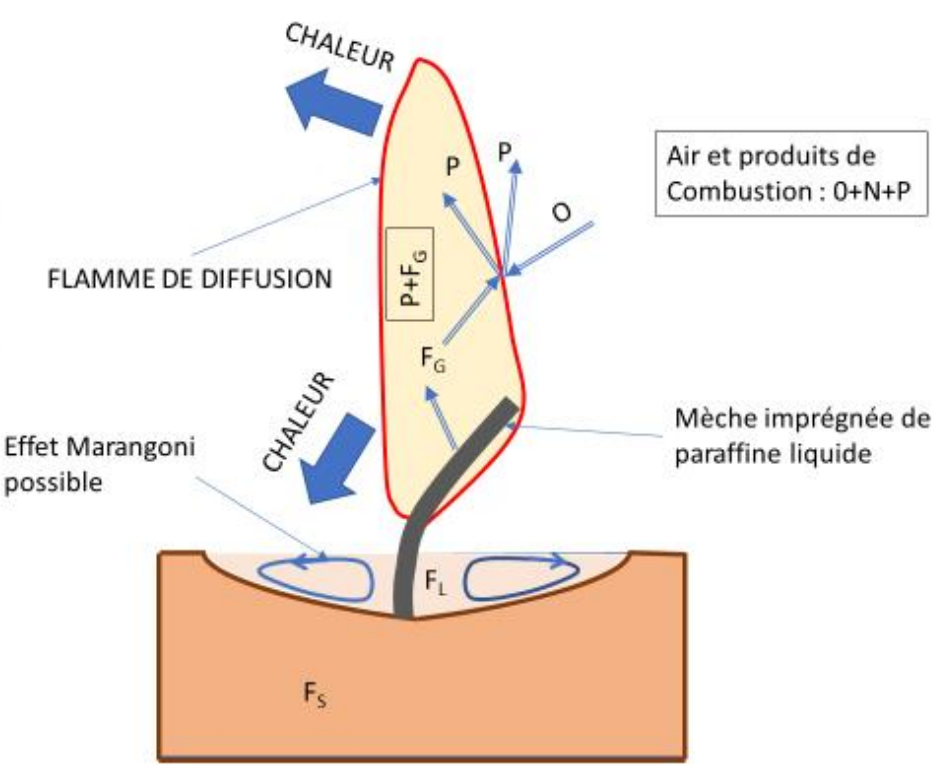

Figure 2. La flamme de bougie, processus complexe comprenant une flamme de diffusion. Le schéma montre en particulier la diffusion des espèces de part et d'autre de la surface de flamme et la chaleur qui sert à la fusion de la paraffine, ainsi que l'agitation du liquide rendue possible par l'effet Marangoni'.

La flamme de diffusion se développe donc à la séparation entre une zone riche en oxydant et une autre riche en réducteur, où les concentrations sont dans un rapport optimum pour la réaction (le rapport stochiométrique pour une combustion monoréactive). La dénomination provient du fait que la persistance de ce type de flamme est conditionnée par la diffusion des réactants à travers le mélange des gaz brûlés de part et d'autre de la surface. Mais l'on préfère parfois la dénomination de flamme non prémélangée au lieu de celle de "flamme de diffusion", car le phénomène de diffusion n'est pas propre à ces flammes et est aussi présent dans les flammes de prémélange même s'il est localisé autrement comme c'est expliqué plus loin en section 4.

L'intérieur de la flamme est chauffé essentiellement par rayonnement à partir de la zone de réaction en surface. Ce chauffage à l'abri de l'oxygène provoque la décomposition de vapeurs organiques sous forme de suies constituées principalement de carbone. Ce sont ces suies chauffées à blanc (autour de $1500^{\circ} \mathrm{C}$ ) qui donnent la couleur de la flamme, et permettent donc à la bougie de nous éclairer.

\footnotetext{
${ }^{6}$ http://www.encyclopedie-environnement.org/physique/diffusion-etape-ultime-bon-melange/

${ }^{7}$ L'effet Marangoni, du nom d'un scientifique Italien, est dû à l'inhomogénéité de la température de la surface chauffée causant des variations de tension de surface qui induisent à leur tour des mouvements du liquide entrainé par viscosité. 


\subsection{Le problème de Burke-Schumann}

Seule la résolution des équations qui régissent la dynamique des fluides réactifs permet de prédire théoriquement la structure des flammes. La résolution est généralement numérique, mais il existe des cas simplifiés où le calcul analytique donne des résultats.

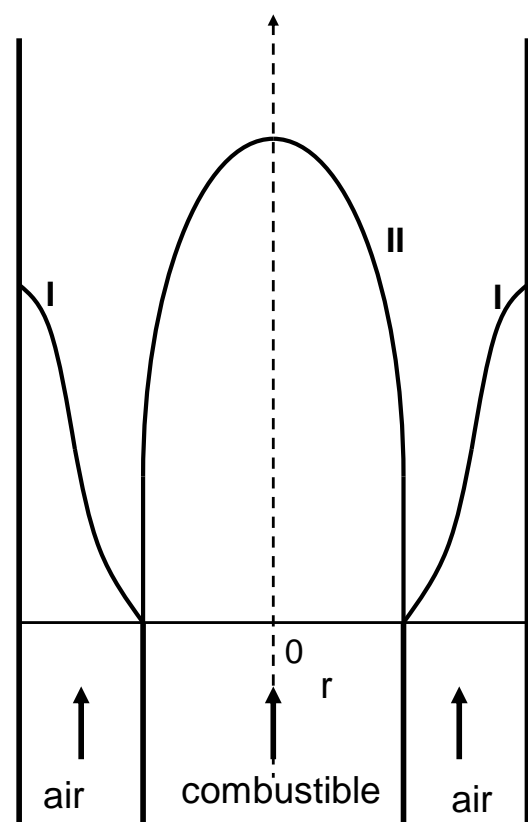

a)

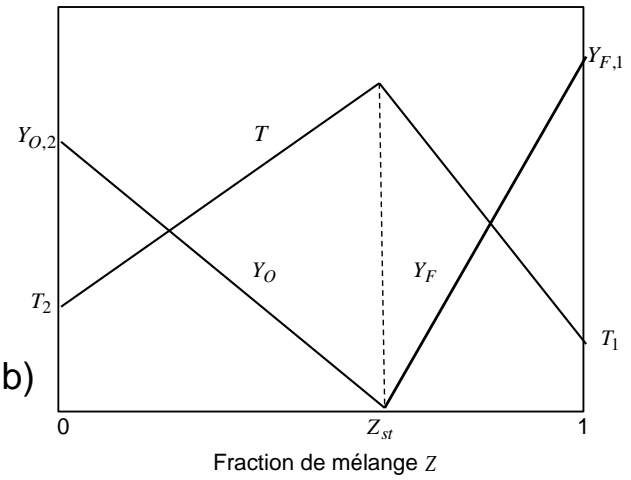

c)

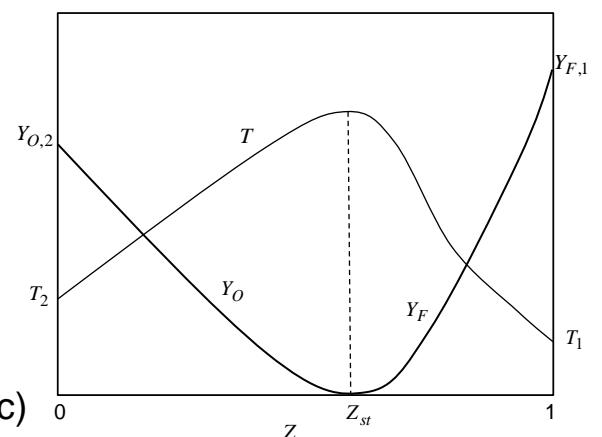

Figure 3. a) Formes de la flamme de diffusion du type Burke-Schumann pour des cylindres coaxiaux. Deux exemples sont donnés sur la même figure : I) sous-ventilée II) sur-ventilée ; b) Solution en Z correspondant à la théorie dans l'hypothèse où la flamme est supposée mince;c) Allure d'une solution en Z réelle.

C'est ce qui se produit avec l'exemple qui suit où le système, plus simple que la bougie, consiste à injecter directement le combustible et le comburant séparément. Des hypothèses simplificatrices, comme celle de la flamme mince en écoulement laminaire et stationnaire, permettent de déterminer la position de la flamme et le champ de température et des concentrations. Le problème peut être traité avec deux cylindres concentriques de sections circulaires : on injecte le combustible $F$ par le cylindre intérieur et le comburant $O$ entre les deux cylindres. C'est alors le problème de Burke-Schumann'.

Ce problème de flamme laminaire, entièrement gouverné par la diffusion des espèces, se résout analytiquement. La flamme supposée mince se positionne à l'endroit où la concentration est stœchiométrique. On néglige en particulier la diffusion des espèces dans la direction axiale devant la diffusion radiale et on fait certaines hypothèses simplificatrices dites de Shvab-Zeldovich suivantes :

- écoulement stationnaire - pas de thermo-diffusion $\mathrm{D}_{\mathrm{T}}=0$ •forces extérieures à distance négligeables • viscosité négligeable $\bullet$ pression statique constante en première approximation $\bullet$ loi de Fourier pour la conduction thermique $\bullet$ loi de Fick pour la diffusion, et un seul coefficient de diffusion pour toutes les espèces $\bullet$ le nombre de Lewis est proche de l'unité $\bullet$ une seule réaction $\bullet$ mélange de $\mathrm{N}$ gaz parfaits.

La solution s'exprime en série de fonctions de Bessel en se limitant aux deux premiers termes, ce qui explique les formes de flamme peu réalistes de la figure $3 \mathrm{a}$, mais permet de bien différencier les cas I où le débit d'oxygène injecté est faible devant celui du fioul, la flamme est alors ouverte (en forme de tulipe) et le cas II où le débit d'oxygène est suffisant pour que la flamme se referme dans le cylindre intérieur. 
On repère la position de la flamme mince dans le diagramme de la figure $3 b$ donnant les fractions massiques des réactants et la température locales en fonction de la fraction de mélange $Z$ : les profils de température et de concentration sont en segments rectilignes avec un angle au niveau de la fraction de mélange stœchiométrique $Z=Z_{s t}$. La fraction de mélange $Z$ est : $Z=\left(r_{s t} Y_{F}-Y_{O}+Y_{O, 2}\right) /\left(r_{s t} Y_{F, I}+Y_{O, 2}\right)$, où $r_{s t}=v^{\prime}{ }_{O} \mathscr{M}_{O} / v^{\prime}{ }_{F} \mathscr{M}_{F}$ est le rapport stoechiométrique pour la réaction chimique $: v_{F}^{\prime} F+v_{O}{ }_{O} O \rightarrow P$, avec les coefficients stœchiométriques $v_{F}^{\prime}$ et $v^{\prime}{ }_{O}$ du premier membre et les masses molaires respectives $\mathscr{M}_{F}, \mathscr{M}_{O}$ des réactants, les indices 1 et 2 correspondant aux conduits d'alimentation en $F$ et en $O$ respectivement.

La figure $3 \mathrm{c}$ donne les résultats obtenus numériquement pour une flamme épaisse : les profils angulaires sont alors émoussés.

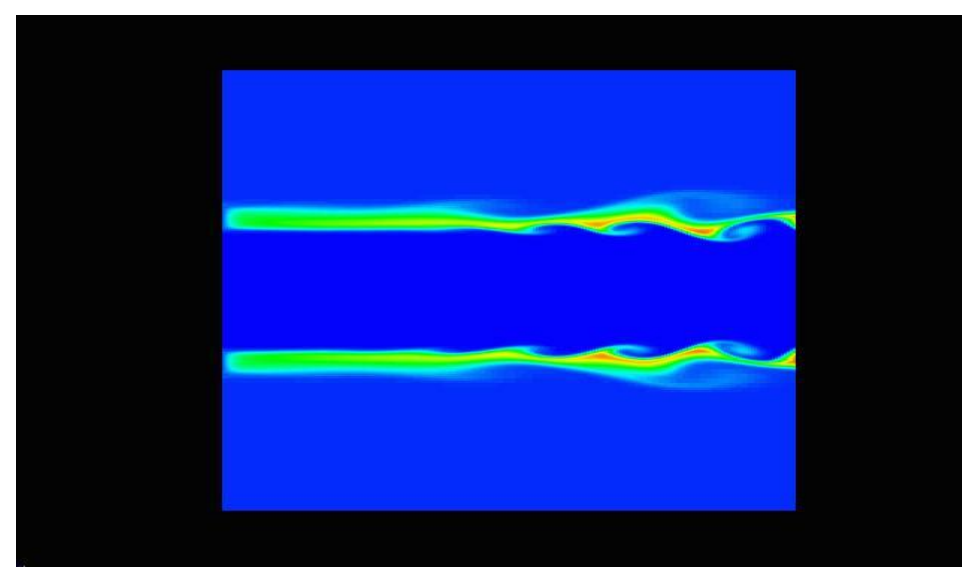

Figure 4. Double instabilité de Kelvin-Helmholtz en aval d'un injecteur $\mathrm{O}_{2}-\mathrm{H}_{2}$ allumé. C. Nicoli, P. Haldenwang, 2002.

Le calcul numérique - comme le montre la figure 4 tirée d'une vidéo aimablement fournie par les auteurs cités - permet de déterminer des profils de flammes de diffusion laminaires plus complexes apparaissant lorsque la vitesse du jet central diffère de celle du jet extérieur.

\section{Combustion non prémélangée turbulente}

La turbulence accélère la diffusion des espèces chimiques et de la chaleur, comme le démontre René Moreau dans l'encyclopédie de l'Environnement ${ }^{8}$, et elle favorise donc la chimie de la combustion. Si la chaleur diffuse mieux, la température sera plus élevée dans la zone réactive et la réaction chimique sera d'autant plus rapide. Mais elle a comme autre effet celui de déformer la surface des flammes par plissement et étirement. Les nombreux mouvements tourbillonnaires présents, jusqu'à l'échelle des plus petits tourbillons, augmentent l'aire de contact entre comburant et combustible. Cela contribue efficacement à accroître la vitesse de combustion globale.

La figure 5 montre la variété d'échelles de longueur correspondant à des tailles de tourbillons susceptibles d'interagir avec la flamme de diffusion lors de l'injection de combustible dans une atmosphère de comburant. 


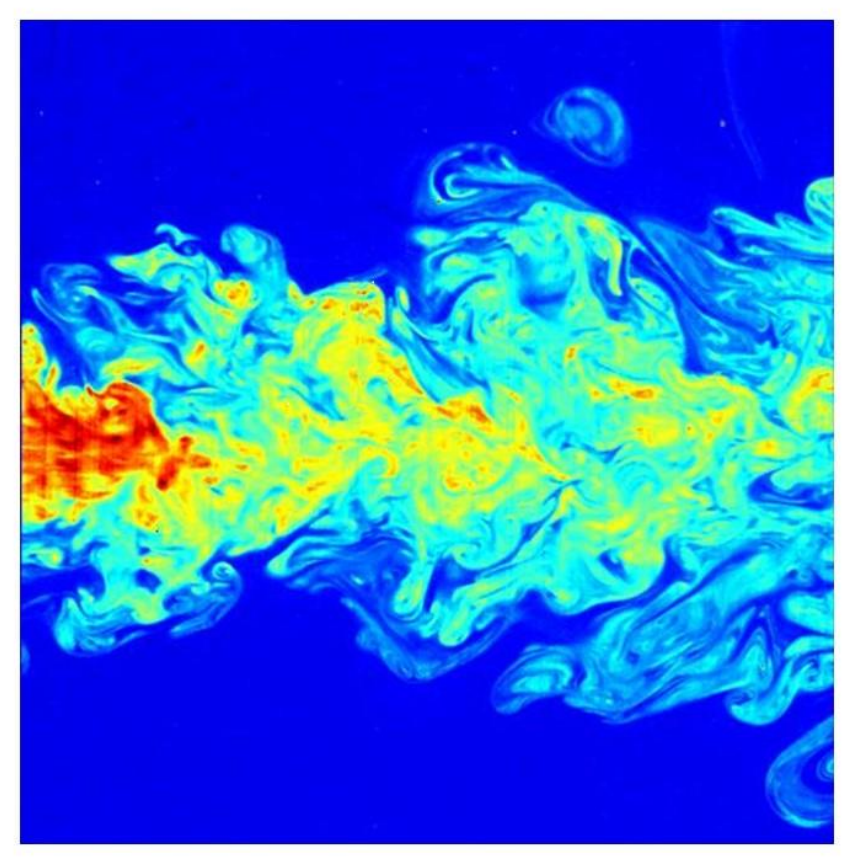

Figure 5. Visualisation d'un jet turbulent réalisée par fluorescence induite par laser. Le jet présente une large gamme d'échelles de longueur représentées par des couleurs différentes, caractéristique importante des écoulements turbulents. http://en.wikipedia.org/wiki/Turbulence

Pour caractériser le régime de combustion turbulente on se doit de comparer la taille des tourbillons à l'épaisseur de la flamme. La classification des régimes turbulents (voir la figure 6, schématisant le diagramme de Peters ${ }^{\mathrm{ii}}$ ) est difficile en combustion non prémélangée du fait qu'il n'y a pas de définition simple de l'épaisseur de flamme.

Les échelles turbulentes sont évaluées partiellement dans l'espace de la fraction de mélange $Z$. Nous avons :

- Une épaisseur de flamme $\ell_{D}=\left(D_{s t} / a\right)^{1 / 2}$. Le coefficient de diffusion à l'emplacement de la flamme, lorsque $Z=Z_{\text {st }}$ avec taux d'étirement $a$.

- L'épaisseur de diffusion correspondante dans l'espace des fractions de mélange : $Z_{f}=\ell_{D}|\nabla Z|_{s t}$. L'épaisseur de diffusion peut être réécrite en fonction du taux d'étirement $a$ et d'une valeur instantanée du "taux de dissipation scalaire" local $\chi_{s t}=2 D_{s t}|\nabla Z|_{\mathrm{st}}{ }^{2}$. Ainsi, nous avons $\Delta Z_{f}=\left(\chi_{s t} / 2 a\right)^{1 / 2}$.

- Une épaisseur de réaction $\Delta Z_{R}=\varepsilon \Delta Z_{f}$, égale à l'épaisseur de la couche d'oxydation, qui est proportionnelle à $\chi_{s t}^{1 / 4}$.

La figure 6, où $Z$ ' est la fluctuation moyenne de fraction de mélange, donne un exemple de combustion non prémélangée méthane-air avec les quatre réactions chimiques :

1) $\mathrm{CH}_{4}+2 \mathrm{H}+\mathrm{H}_{2} \mathrm{O}=\mathrm{CO}+4 \mathrm{H}_{2} \mathrm{O}$, 2) $\left.\mathrm{CO}+\mathrm{H}_{2} \mathrm{O} \rightarrow \mathrm{CO}_{2}+\mathrm{H}_{2}, 3\right) \mathrm{H}+\mathrm{H}+\mathrm{M} \rightarrow \mathrm{H}_{2}+\mathrm{M}$, 4) $\mathrm{O}_{2}+3 \mathrm{H}_{2} \longrightarrow 2 \mathrm{H}+2 \mathrm{H}_{2} \mathrm{O}$. 


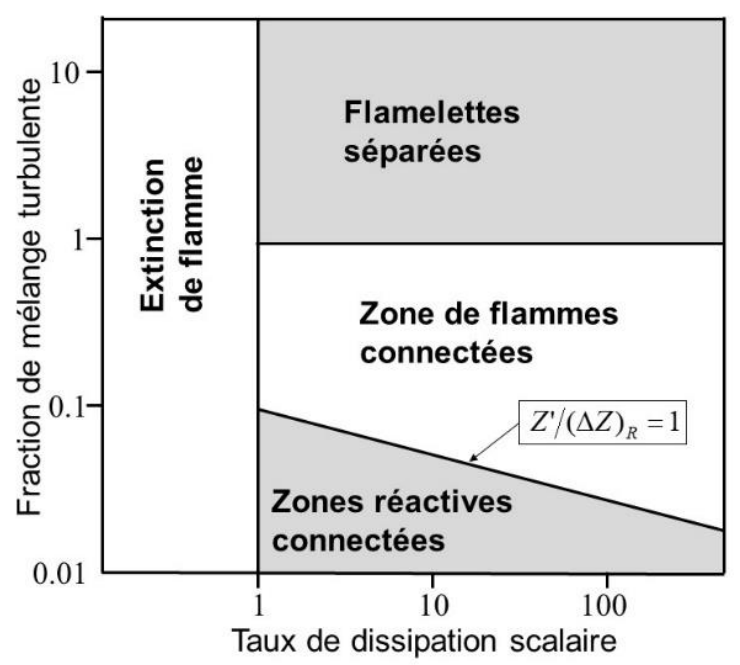

Figure 6. Représentation simplifiée du diagramme de Peters (réf. ii) dans le plan « taux de dissipation $\chi d \widetilde{\chi \text { st }^{-}}$fraction de mélange turbulente $Z_{s t}^{\prime}(\Delta Z) F$ » pour les régimes de combustion non prémélangée méthane-air.

Dans le diagramme on voit quatre zones dont l'une fait apparaitre des flamelettes.

Le concept de flamelette, découvert par Williams ${ }^{\text {iii }}$, est actuellement utilisé dans tous les types de combustion turbulente (mentionnons également Peters, Kuznetsov et Bray pour les extensions de ce concept). Les flamelettes sont de minces couches réactives-diffusives incorporées dans un champ d'écoulement turbulent non réactif. Poinsot et Veynante ${ }^{\mathrm{iv}}$ donnent une définition moins restrictive du régime de flamelettes qui correspond à un front de flamme continu sans désactivation et où aucune référence n'est faite aux flammes minces.

Précisons que la désactivation de la flamme (en anglais "flame quenching") se produit lorsqu'un front de flamme est soumis à des perturbations externes, telles que des pertes de chaleur ou un étirement aérodynamique, suffisamment fortes pour réduire le taux de réaction à une valeur négligeable et, dans certains cas, pour supprimer complètement la vitesse de réaction.

On constate qu'il n'y pas de flamme dans la partie gauche du diagramme de Peters où le taux de dissipation scalaire est inférieur à la valeur stœchiométrique, c'est-à-dire à 1. Dans la partie droite, audessus de 1 la flamme est allumée ; elle se déforme de plus en plus lorsque la turbulence augmente, d'abord par plissement et étirement, puis la zone réactive se morcelle et la flamme entière finit par se déchirer en flamelettes séparées aux fractions de mélange turbulente $Z^{\prime}{ }_{\mathrm{st}} /(\Delta Z)_{\mathrm{F}}$ supérieures à l'unité.

Les flammes issues d'injecteurs dans les moteurs ${ }^{\mathrm{v}}$ ont été les plus étudiées, tant expérimentalement que numériquement. On déduit du diagramme de la figure 6 des renseignements sur la morphologie des flammes. Cela est important pour choisir le bon modèle de combustion turbulente qui permettra de traiter numériquement les écoulements. On est souvent amené à considérer plusieurs régions et de traiter chacune d'elle avec une méthode différente.

Les modèles de résolution numérique de la combustion diffèrent selon que la combustion relève ou non d'un prémélange. Le lecteur intéressé pourra consulter aussi les ouvrages spécialisés déjà cités (réf. ii, iii, iv). 


\section{Combustion prémélangée}
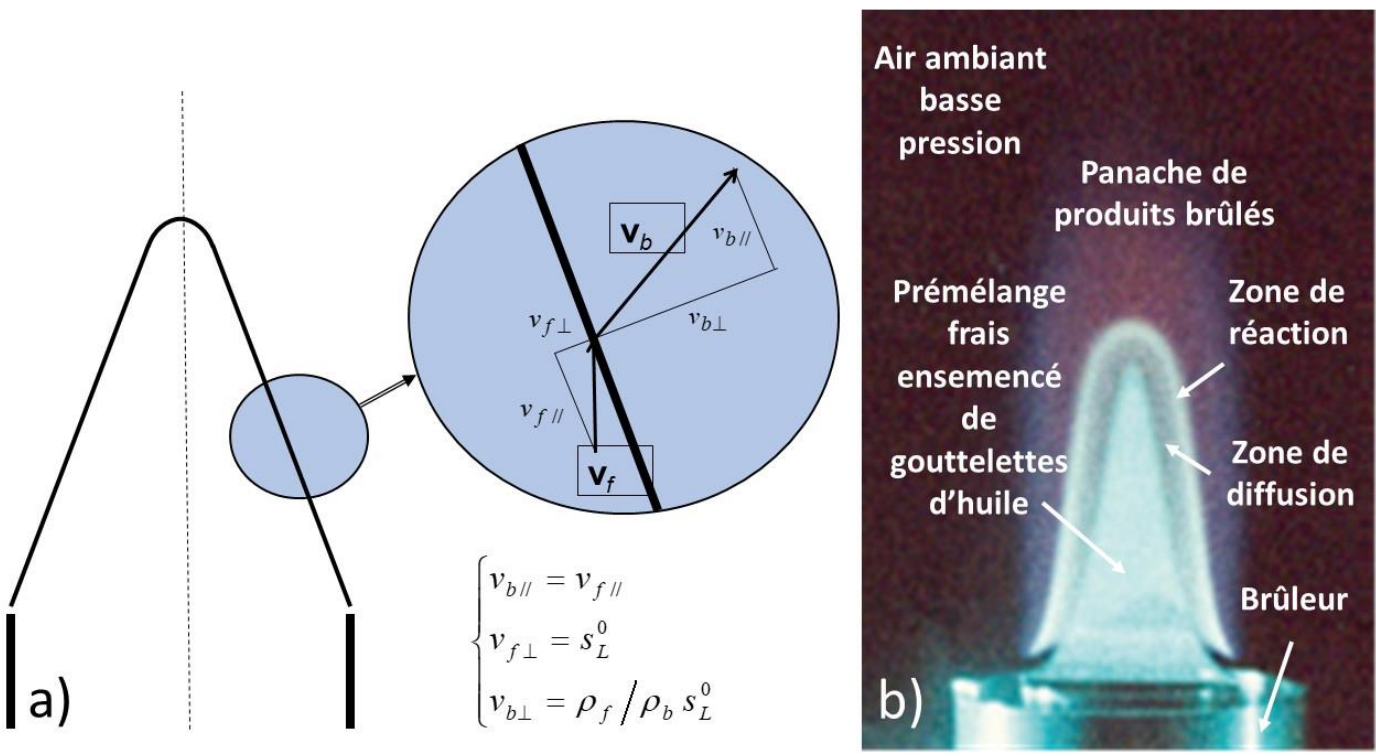

Figure 7. a) Application de la théorie de Rankine-Hugoniot (voir Annexe 1) à la flamme oblique issue d'un bec Bunsen : composition des vitesses de part et d'autre du front de flamme. Les gaz brûlés et frais sont désignés respectivement par les indices $b$ et $f$. b) Photographie sous plan laser d'une flamme de bec Bunsen sous basse pression - ce qui épaissit sa structure interne - visible grâce à l'ensemencement par de fines gouttelettes d'huile.

Comme nous l'avons vu la combustion est fortement contrainte par la nécessité de mélanger combustible et comburant. Un prémélange augmente considérablement l'efficacité. On passe d'un cas à l'autre dans un simple bec Bunsen en positionnant la virole, soit pour permettre l'entrée de l'air en amont et donc le prémélange, soit en l'obturant ce qui conduit à la flamme de diffusion. Dans une cuisinière à gaz, le prémélange est obtenu grâce à la conception du brûleur qui permet l'injection d'air. Si on l'allume sans le dessus du brûleur, on obtient une grande flamme moins efficace thermiquement et source de suies à cause de la combustion incomplète.

Nous avons vu qu'une flamme de diffusion se positionne au contact entre une zone comburante et une zone combustible. Elle est en quelque sorte un lieu géométrique de points à concentration stœechiométrique.

Dans un prémélange homogène au contraire, la position de la flamme n'est pas déterminée a priori. Le phénomène qui se développe est une onde résultant d'interactions mettant en jeu convection, diffusion de la chaleur et des espèces et réaction chimique avec son énergie d'activation $E_{a}$ et son énergie libérée $\Delta \mathrm{h}$. Dans un gaz non réactif les seules ondes de discontinuité sont des ondes de compression appelées ondes de choc, qui ont la propriété d'avoir une vitesse supersonique par rapport à l'écoulement amont. Les ondes de détente, telles que les ondes centrées existent bien mais elles sont continues. 


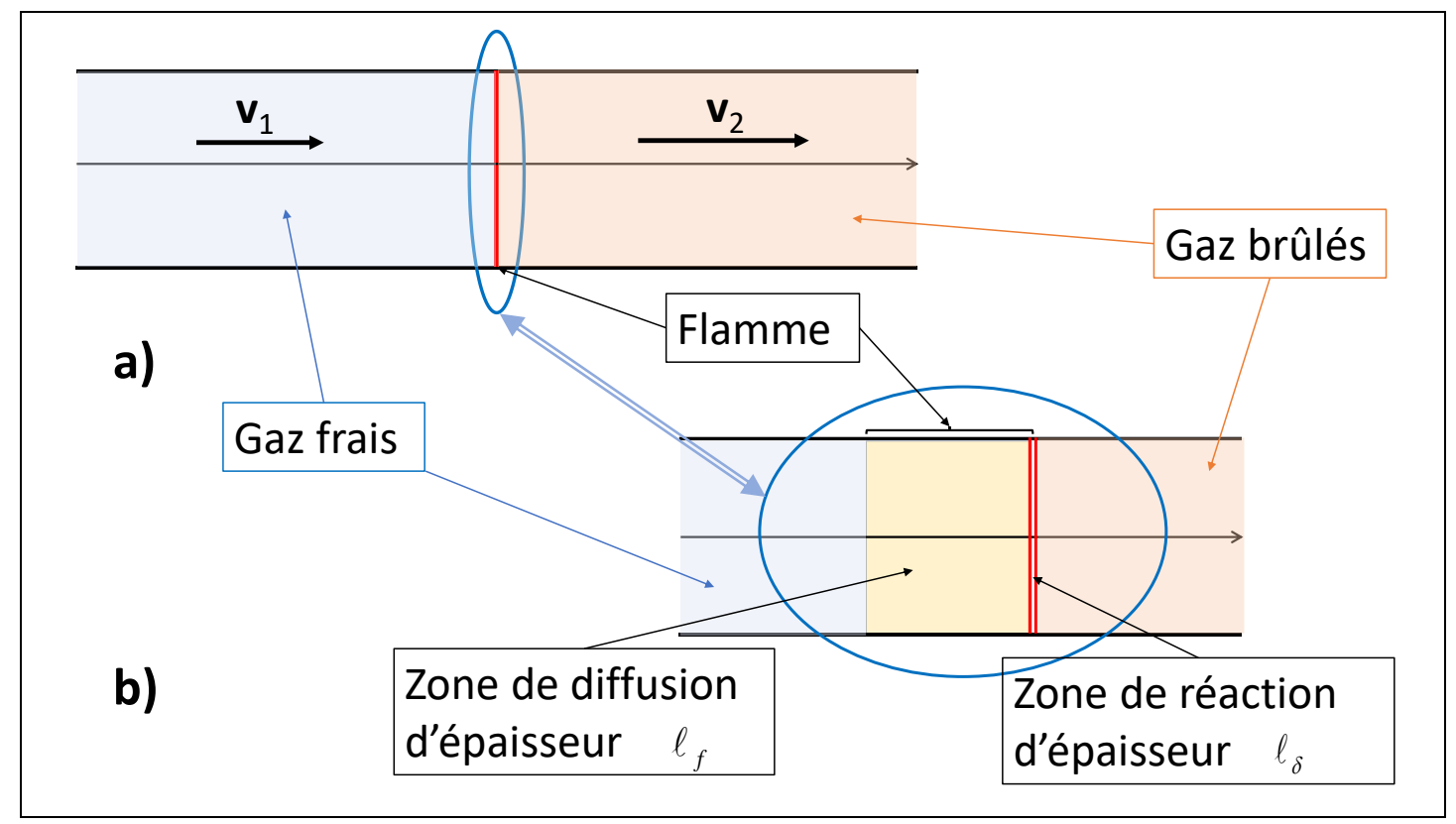

Figure 8. Deux échelles pour un même problème : a) Configuration de Rankine-Hugoniot : représentation du mouvement stationnaire à l'échelle du laboratoire dans un système lié à l'onde ; tuyauterie droite adiabatique remplie initialement d'un mélange de comburant et de combustible, $v_{1}$ est la vitesse de combustion laminaire adiabatique généralement notée $s_{L}{ }^{0}$ b) Structure de flamme mince de prémélange laminaire dans la conduite rectiligne à petite échelle : la flamme proprement dite d'épaisseur $\ell_{f}$ présente une zone de diffusion-convection et une zone de réaction-diffusion d'épaisseur $\ell_{\delta}$. Ici l'énergie d'activation de la réaction est très grande et l'on

vérifie $\ell_{\delta}<\ell_{f}$.

En revanche, dans un mélange gazeux réactif peuvent se développer pour les mêmes concentrations, deux types d'onde de discontinuité. L'onde de compression existe comme dans le cas non réactif : c'est une onde de choc boostée par l'énergie de la réaction chimique, donc plus intense et tout à fait supersonique (2000 à $3000 \mathrm{~m} / \mathrm{s}$ ), que l'on nomme détonation. Mais les lois de la thermodynamique rendent possible l'existence d'une onde de détente discontinue appelée aussi onde de déflagration - ou flamme de prémélange - qui est nettement subsonique (de l'ordre du $\mathrm{m} / \mathrm{s}$ ). La forme de la flamme produite en sortie d'un bec Bunsen résulte d'un équilibre entre la vitesse de sortie du gaz et la vitesse de propagation de la flamme vers l'intérieur (voir Figure 7). Cette propagation d'une flamme de prémélange est théoriquement abordée dans le cas simplifié d'un tube rectiligne.

\subsection{Propagation d'une flamme prémélangée}

La théorie simplifiée de Rankine-Hugoniot ${ }^{\mathrm{vi}}$ (voir Annexe 1) rend bien compte des différentes ondes de combustion planes laminaires susceptibles de se présenter dans une tuyauterie droite adiabatique (c'est-à-dire n'échangeant pas de chaleur avec l'extérieur) remplie initialement d'un mélange de comburant et de combustible. L'allumage à l'une des extrémités du tube au moyen d'une étincelle permet d'abord la progression dans les gaz frais d'une onde lente à vitesse subsonique de l'ordre du $\mathrm{m} / \mathrm{sec}$ - dite de déflagration - qui n'est rien d'autre qu'une flamme plane de prémélange. L'ordre de grandeur de la vitesse de propagation de cette flamme est le $\mathrm{m} / \mathrm{s}$. Puis au cours du temps, cette onde s'accélère et devient une onde de détonation très rapide progressant à vitesse supersonique, de l'ordre de plusieurs $\mathrm{km} / \mathrm{sec}$.

Nous avons considéré pour ce système unidimensionnel une origine liée à l'onde comme indiqué sur la figure 8a avec une zone amont $(x<0)$ contenant les gaz frais de vitesse constante, séparée par une surface de discontinuité fixe en $x=0$, de la zone aval contenant les gaz brûlés de vitesse constante. Les équations à la discontinuité ont été écrites, successivement pour la masse, la quantité de mouvement et l'énergie, ainsi que les équations d'état du mélange gazeux. Après simplification ont été obtenues 
les relations de Rankine-Hugoniot : $(p-1) /(\vartheta-1)=-\gamma M_{1}^{2}$ (droite de débit) et $p=[(1-\gamma) \vartheta+1+\gamma+2 \gamma \tau]$ $/[(1+\gamma) \vartheta+1-\gamma]$ (adiabatique d'Hugoniot).

Dans cette théorie simplifiée, les points solutions ont ainsi pu être obtenus graphiquement par l'intersection d'une courbe et d'une droite dans le plan volume-pression dans les deux cas : la déflagration pour $\mathrm{M}_{1}<1$ et la détonation pour $\mathrm{M}_{1}>1$, le point de fonctionnement se trouvant à l'intersection de la droite de débit et de l'adiabatique d'Hugoniot qui est de forme hyperbolique.

L'onde de déflagration de vitesse $\mathrm{v}_{1}=s_{L}{ }^{0}$ correspond à un point de la branche $\mathrm{F}_{1} \mathrm{~F}$ où la variation de pression est faible et où l'augmentation du volume massique indique une forte dilatation. Mais trouver la position exacte de ce point n'est pas immédiat. Nous expliquons sa détermination à la section 4.2.

Le point de détonation sur la branche $\mathrm{T}_{1} \mathrm{~T}_{2}$ est plus facile à déterminer.

L'onde de détonation se produit au point de Chapman-Jouguet seul point stable de l'adiabatique d'Hugoniot. L'évolution de la production d'entropie réduite $\left(\mathrm{s}_{2}-\mathrm{s}_{1}\right) / \mathrm{c}_{\mathrm{v}}$ à la traversée de l'onde en fonction de la masse volumique réduite $\rho_{1} / \rho_{2}$ présente en effet un minimum en ce seul point où les paramètres sont facilement calculables analytiquement. Remarquons seulement que sa vitesse est déterminée par les conditions au point $\mathrm{T}$ où la droite de débit est tangente à la courbe d'Hugoniot. Les coordonnées du point $\mathrm{T}$ sont aisément déductibles par un simple calcul analytique.

\subsection{La vitesse de combustion de la flamme plane adiabatique de prémélange}

On sait assez bien déterminer les paramètres d'une onde de déflagration par des calculs analytiques dans le cas monoréactifs et plus aisément par le calcul numérique. D'autre part les expériences menées avec le bec Bunsen conduisent à de bons résultats puisque le long des arêtes du cône (voir la figure 7a) la vitesse normale est assimilable à la vitesse de combustion de la flamme plane en négligeant les effets de courbure. La photo $7 \mathrm{~b}$ réalisée sous une pression de $1000 \mathrm{~Pa}$ met en évidence les mêmes trois zones que la flamme plane observée à petite échelle de la figure $8 \mathrm{~b}$.

Mais il est possible d'évaluer comme l'a fait Williams (réf. iii) la vitesse de combustion $\mathrm{s}_{\mathrm{L}}^{0}$ par des raisonnements - et des calculs élémentaires - portant sur les quantités fondamentales qui régissent la physique de l'onde de déflagration. Rappelons pour cela la liste des grandeurs de base qui interviennent dans le processus. Il y a successivement : l'épaisseur de la flamme assimilable à celle de la zone de diffusion $\ell_{\mathrm{f}}$ de la figure $8 \mathrm{~b}$, la vitesse du mélange frais $\mathrm{v}_{\mathrm{l}}=\mathrm{u}_{1}=\mathrm{s}_{\mathrm{L}}{ }^{0}$, la chaleur de réaction $\Delta \mathrm{h}$ (énergie libérée par unité de masse du mélange de réactifs), le taux de réaction $\dot{\mathrm{W}}$ (masse du mélange de réactifs convertie en produit de réaction par unité de volume par seconde).

On peut évaluer le gradient thermique dans la zone de diffusion par la relation $\mathrm{dT} / \mathrm{dx} \sim\left(\mathrm{T}_{2}-\mathrm{T}_{1}\right) / \ell_{\mathrm{f}}$, en considérant que la température des gaz frais $T_{1}$ est uniforme, et que la température des gaz brûlés $T_{2}$ est également uniforme puisque les parois du tube ne laissent pas passer la chaleur. La conservation de l'énergie donne par ailleurs $\Delta h=c_{p}\left(T_{2}-T_{1}\right)$.

Pour que le processus soit stationnaire, il faut que l'énergie libérée par unité de volume du mélange réactif soit égale à l'énergie conduite par unité d'aire, ce qui donne :

$\Delta \mathrm{h} \dot{\mathrm{W}} \ell_{\mathrm{f}} \approx \lambda\left(\mathrm{T}_{2}-\mathrm{T}_{1}\right) / \ell_{\mathrm{f}}$. Stationnarité implique aussi conservation du débit : $\rho_{1} \mathrm{u}_{1}=\dot{\mathrm{W}} \ell_{\mathrm{f}}$.

On déduit plusieurs relations intéressantes. D'abord l'épaisseur de flamme s'évalue comme

$\ell_{\mathrm{f}} \approx(\lambda / \mathrm{cp} \dot{\mathrm{W}})^{1 / 2}$.

La vitesse de combustion s'écrit

$\mathrm{u}_{1} \approx\left(1 / \rho_{1}\right)(\lambda \dot{\mathrm{W}} / \mathrm{cp})^{1 / 2}$ 
Pour les prémélanges avec l'air, les vitesses mesurées varient par exemple entre $0,845 \mathrm{~m} / \mathrm{sec}$ avec le méthane et $1,845 \mathrm{~m} / \mathrm{sec}$ avec l'éthylène (valeurs fournies dans l'Encyclopédie Universalis). Avec des valeurs représentatives pour $\lambda$, $\mathrm{c}_{\mathrm{p}}$ et $\rho_{0}$, et avec $\mathrm{v}_{0} \approx 50 \mathrm{~cm} / \mathrm{s}$, l'équation (4) donne $\ell_{\mathrm{f}} \sim 10^{-2} \mathrm{~cm}$. Comme le fait remarquer Williams, par conséquent, $\ell_{\mathrm{f}}$ est grand comparé à un libre parcours moléculaire moyen (environ $10^{-5} \mathrm{~cm}$ ), et les équations du continuum de la dynamique des fluides sont valables dans l'onde de déflagration; mais $\ell_{\mathrm{f}}$ est petit comparé aux dimensions typiques de l'équipement expérimental (par exemple, le diamètre de l'embouchure du brûleur, et donc le rayon de courbure du cône de flamme, pour les expériences avec des brûleurs de type Bunsen), et les ondes de déflagration laminaires peuvent être approchées comme des discontinuités dans de nombreuses expériences.

Dixit F.A. Williams, en principe, ces résultats devraient fournir une estimation numérique de $\mathrm{u}_{1}$. Cependant, dans la pratique, les valeurs de $\dot{\mathrm{W}}$ sont tellement incertaines que l'équation (2) est plus utile pour estimer $\dot{\mathrm{W}}$ à partir des valeurs expérimentales de $\mathrm{v}_{0}$. Une manière utile de déduire un ordre et une énergie d'activation globale d'une réaction consiste à mesurer les dépendances en $\mathrm{T}$ et $\mathrm{p}$ de $\mathrm{v}_{0}$ et à les corréler empiriquement avec l'équation suivante en ajustant $n$ et $E_{1}$.

En effet, si l'on admet pour le taux de réaction $\dot{\mathrm{W}} \approx \mathrm{p}^{\mathrm{n}} \mathrm{e}^{-\mathrm{E} / \mathrm{RT}}{ }_{\mathrm{a}}$, où $\mathrm{n}$ est l'ordre de la réaction chimique ${ }^{9}$, c'est-à-dire le nombre de moles des réactants intervenant dans le premier membre - par exemple $\mathrm{n}=2$ pour la réaction $\mathrm{A}+\mathrm{B} \rightarrow \mathrm{P}$ ou $\mathrm{n}=v$ pour $v \mathrm{~A} \rightarrow \mathrm{P}-$ on trouve :

$$
\mathrm{u}_{1} \approx \mathrm{p}^{(\mathrm{n} / 2)-1} \mathrm{e}_{\mathrm{a}}^{-\mathrm{E} / 2 \mathrm{RT}}{ }_{1}
$$

Ces calculs simples permettent en particulier d'évaluer l'influence de la température des gaz brûlés $\mathrm{T}_{1}=\mathrm{T}_{\mathrm{b}}$ sur l'épaisseur $\ell_{\mathrm{f}}$ de la flamme et sur la vitesse de combustion. La pression $\mathrm{p}$ - qui varie très peu à la traversée de la flamme - intervient également.

Mais seule l'étude de la structure de l'onde de déflagration plane schématisée sur la figure $6 \mathrm{~b}$ où l'abscisse $\mathrm{x}$ a été dilatée artificiellement, permet la détermination précise de sa vitesse adiabatique $\mathrm{s}_{\mathrm{L}}{ }^{0}$. Le calcul analytique direct est possible dans des cas simples. Pour faire ce calcul, on fait l'approximation de Shvab-Zel'dovich présentée en section 2.2, qui suppose en particulier un nombre de Lewis $\left(\mathrm{Le}=\lambda / \rho \mathrm{D} \mathrm{c}_{\mathrm{p}}\right)$ unité, ce qui signifie que la diffusion des espèces et celle de la chaleur - c'est-àdire la conduction thermique - ont lieu à la même vitesse. Le détail des calculs ${ }^{\text {vii }}$ ne rentre pas dans le cadre de cet article où nous nous limitons à la présentation des principaux résultats.

Le calcul de cette évolution continue donne $\mathrm{s}_{\mathrm{L}}{ }^{0}=(\mathrm{M} \mathrm{p} / \rho \mathrm{R})\left(\rho \mathrm{DBT}^{\mathrm{s}} / \Lambda\right)^{1 / 2}$, avec $\Lambda$ valeur propre qui assure la continuité de l'évolution au passage de la zone réactive, $\mathrm{B}$ et $\mathrm{s}$ constantes de cinétique chimique définies en section 1 et $\mathrm{M}$ masse molaire moyenne du mélange. 


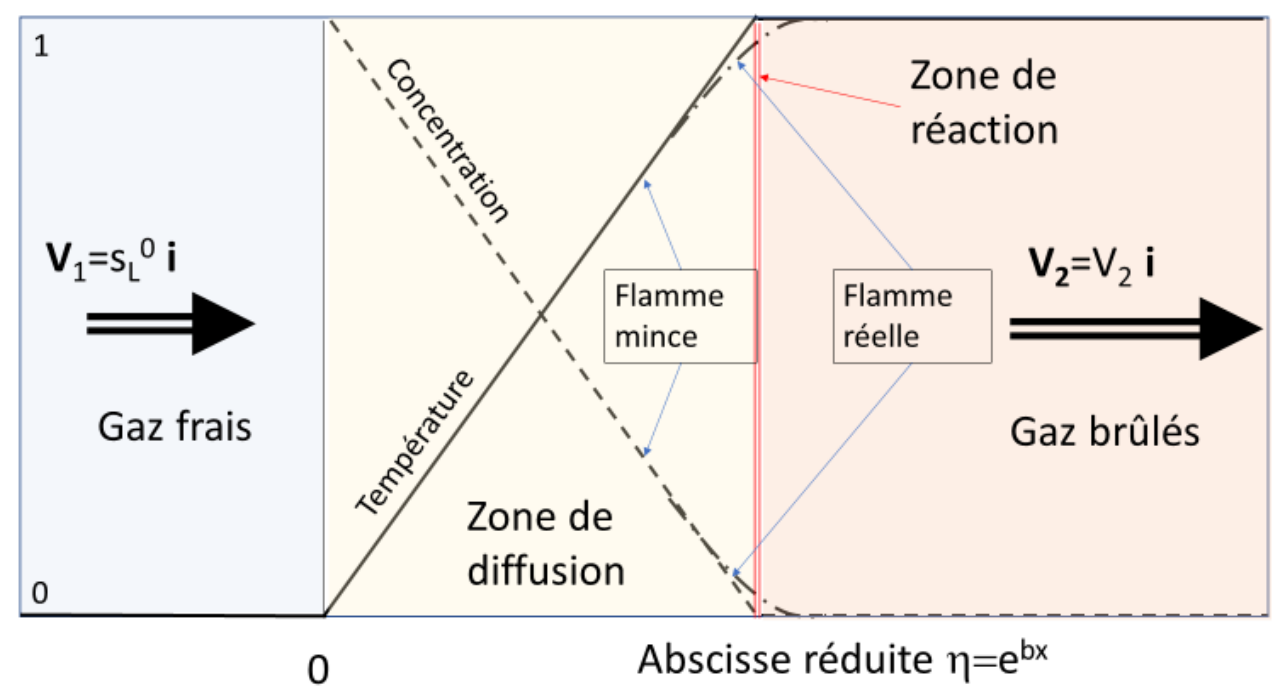

Figure 9. Evolution de la température et de la concentration réduites dans une onde de déflagration en fonction de la variable d'espace $\eta$, avec $b=v_{1} / D$ constant. En traits pleins et en pointillés, les résultats du calcul analytique. En traits mixte, le passage de la zone de réaction pour une flamme réelle.

La figure 9 donne l'évolution de la température et de la concentration réduites en fonction de la variable d'espace (ici l'abscisse réduite). La température réduite est $\beta_{\mathrm{T}}=\left(\mathrm{T}-\mathrm{T}_{1}\right) /\left(\mathrm{T}_{2}-\mathrm{T}_{1}\right)$. Pour une seule réaction $A+B \rightarrow P$ la variable de concentration est $\beta=\mathrm{Y}_{\mathrm{A}} / \mathrm{M}_{\mathrm{A}}=\mathrm{Y}_{\mathrm{B}} / \mathrm{M}_{\mathrm{B}}$.

Les évolutions sont continues dans la zone de diffusion et au passage de la zone réactive où se présente une discontinuité de pente. Cette discontinuité de pente donnant des points anguleux est remplacée par une évolution continue de pente dans le cas de flammes réelles (traits mixtes) où certaines hypothèses restrictives n'ont plus lieu d'être.

Les méthodes analytiques plus modernes conduisent à appliquer de la méthode des développements asymptotiques raccordés à trois échelles : celle de l'écoulement $\ell$, celle de l'épaisseur de diffusion $\ell_{f}$ et celle de la réaction chimique $\ell_{\delta}$ (réf. ii).

Le recours au calcul numérique est indispensable pour les systèmes multiréactifs et si les hypothèses simplificatrices ne sont plus valables.

\subsection{Notions de combustion turbulente prémélangée}

L'action de la turbulence sur les flammes de prémélange a fait l'objet d'études et de modélisation comme ce fut le cas avec les flammes de diffusion. La représentation des régimes de combustion turbulente a lieu dans le plan intensité de la turbulence en fonction de l'échelle de la turbulence. Contrairement aux flammes non prémélangées on compare directement les échelles spatiales de combustion à celles de la turbulence (réf. ii, iv).

Notons que c'est pour les flammes de prémélange que Damköhler ${ }^{\text {viii }}$ a pu le premier présenter des expressions théoriques pour la vitesse de combustion turbulente $\mathrm{s}_{\mathrm{T}}$. 


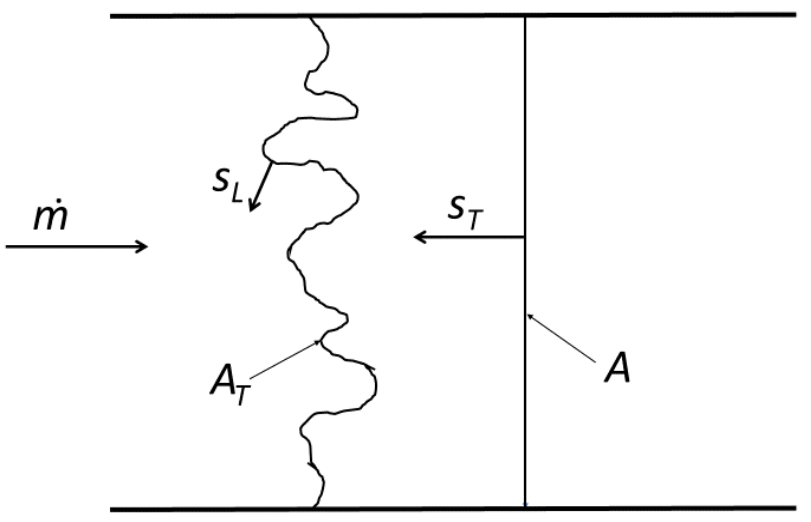

Figure 10. Effet de la turbulence sur une flamme plane mince de prémélange : la relation de Damköhler $\dot{m}=\rho_{f}$ $s_{L} A_{T}=\overline{\rho_{f}} s_{T} A$.

En partant de l'équivalence entre le flux de masse d'un écoulement laminaire à travers la surface de flamme d'aire $\mathrm{A}_{\mathrm{T}}$ et le flux de masse de l'écoulement turbulent à travers la section droite $\mathrm{A}$, il a déduit la relation suivante entre les vitesses de combustion laminaire et turbulente d'une flamme stationnaire dans un tube $: \dot{m}=\rho_{\mathrm{f}} s_{L} A_{T}=\overline{\rho_{\mathrm{f}}} s_{T} A$ où le surlignage indique la moyenne (Figure 10).

\section{Combustion de plaques}

Un exemple classique de combustion hétérogène est fourni par la combustion de plaques, qui présente un grand intérêt pour les études de prévention d'incendie ${ }^{10}$. Cet intérêt a suscité de nombreuses expériences au sol et aussi en micropesanteur où l'une des questions principales est celle de l'émission de suies, très nuisibles dans un habitacle spatial.

La figure 11 donne des exemples de flammes obtenues avec des dispositifs de simulation expérimentale. Les flammes obtenues par combustion du PMMA par exemple ${ }^{i x}$, n'est pas facilement utilisable pour mettre en place les différentes techniques de mesure (à cause de la propagation de la flamme, du temps de changement d'une plaque, etc....). Pour cela, des brûleurs à gaz ont été mis au point pour avoir à disposition une flamme stationnaire allumable et extinguible rapidement. Le brûleur à gaz de la figure 11a est constitué d'un poreux en bronze, d'une épaisseur de $20 \mathrm{~mm}$, en position verticale de taille $400 \mathrm{~mm}$ x $250 \mathrm{~mm}$, fixé devant une chambre de tranquillisation, par lequel les gaz sortent de manière horizontale. Celui des figures $11 \mathrm{~b}$ et c est constitué d'un carré poreux disposé au début de la plaque dans la partie amont, à travers laquelle le combustible gazeux est injecté verticalement à la vitesse $\mathrm{V}_{\mathrm{H}}$ et le comburant circule parallèlement à la vitesse $\mathrm{V}_{\mathrm{O}}$. 


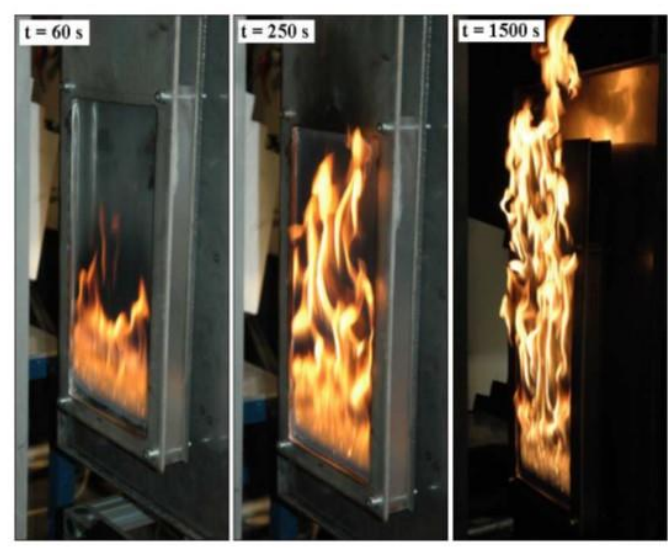

a)

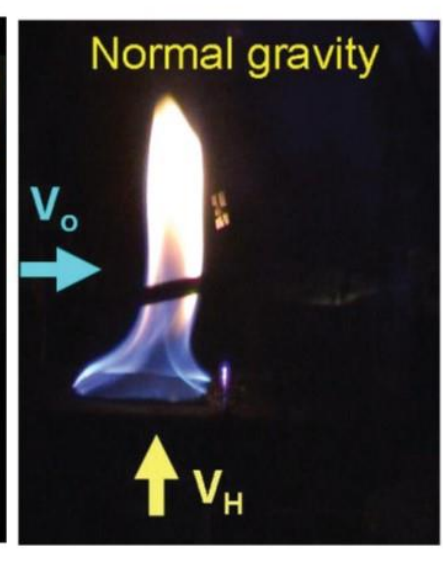

b)

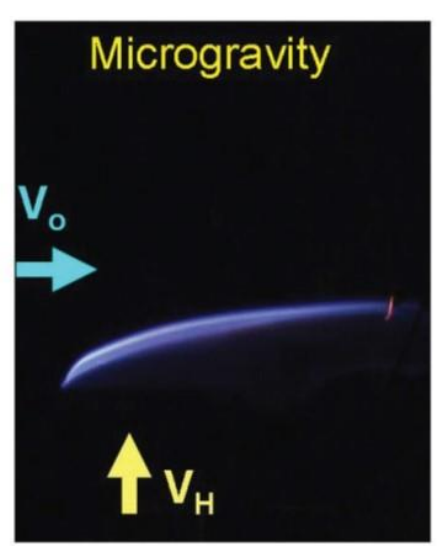

c)

Figure 11. Combustion de plaques au travers de dispositifs de simulation expérimentale : a) Photos évolutives au cours du temps de la combustion d'une plaque verticale de PMMA. (Réf. ix) [Source : @ Autorisation Hebert] ; b) et c) Observations expérimentales d'une flamme de diffusion au-dessus d'une plaque : b) sous gravité normale et c) en microgravité. (G. Legros, communication privée, 2009, réf. $x \mathrm{~V}$ )

Une modélisation simplifiée du problème de la combustion d'une plaque ablative a été fournie par Emmons $^{x}$ : la plaque combustible placée dans un écoulement d'air laminaire à faible nombre de Reynolds fait apparaître après allumage une flamme de diffusion. La chaleur provoque la fusion superficielle du solide et le liquide résultant s'évapore. L'écoulement du fluide est assimilé à celui, très classique en mécanique des fluides, de la couche limite de Blasius au-dessus d'une plaque plane. Cette modélisation analytique du problème de combustion conduit à un profil de flamme mince stationnaire laminaire de forme parabolique. Il est normal que ce résultat ne se vérifie pas au cours des expériences au sol vu les hypothèses simplificatrices utilisées, dont celle consistant à négliger la pesanteur. Mais l'expérience réalisée en micropesanteur montre assez bien un cas de configuration en couche limite d'Emmons (figure 11c)).

Pour ce calcul bidimensionnel stationnaire, on est amené à écrire les bilans de masse totale, de quantité de mouvement, de masse des espèces et de l'énergie. La forme adoptée fait apparaitre le taux de réaction dans le second membre des deux dernières relations. Des bilans de discontinuité sont établis au niveau de la plaque. La discontinuité du bilan de masse met en évidence le débit d'ablation normal $\rho_{\mathrm{s}} \mathrm{v}_{\mathrm{a}}$ qui n'est autre que le débit de combustion et l'effort exercé sur la plaque $\tau_{\mathrm{xy}}$. La vitesse d'ablation s'écrit :

$$
\mathrm{v}_{\mathrm{a}}=-\left(\mathrm{U}_{\infty} / 2 \xi\right)^{1 / 2} \mathrm{f}(0)(\rho \mu)_{\mathrm{P}} / \rho_{\mathrm{H}}
$$




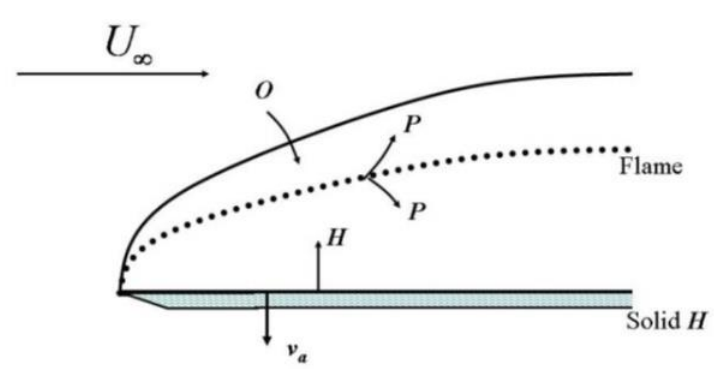

a)

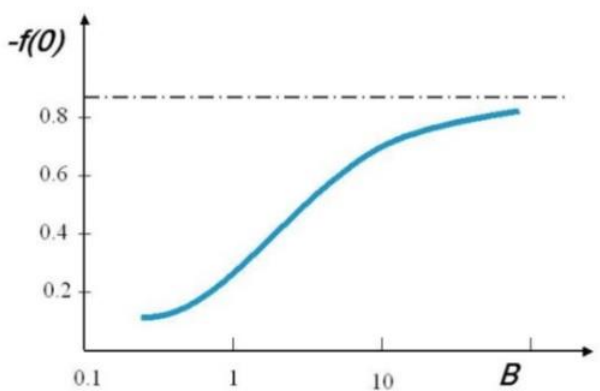

b)

Figure 12. a) La configuration du problème d'Emmons, b) Courbe $(-f(0))$ en fonction de $B$.

Dans cette expression la fonction $f(\xi)$ vérifie l'équation de Blasius classique $f^{\prime \prime \prime}+f f^{\prime \prime}=0$, avec des conditions aux limites $\mathrm{f}^{\prime}(\infty)=1, \mathrm{f}^{\prime}(0)=0$, et $\mathrm{f}(0)=-\mathrm{B} \mathrm{f}^{\prime \prime}(0)$, où $\mathrm{f}=\mathrm{f}(\xi)$ et où intervient le paramètre de Spalding $\mathrm{B}=\left(\beta_{\mathrm{T} \infty}-\beta_{\mathrm{TP}}-\beta_{\mathrm{O} \infty}\right) \Delta \mathrm{h} / \mathrm{l}, \Delta \mathrm{h}$ et 1 étant respectivement l'énergie de la réaction chimique et la chaleur de changement de phase (dans le cas classique non réactif $\mathrm{B}=0$ ). D'autre part la réaction chimique étant $\mathrm{H}+\mathrm{O} \rightarrow \mathrm{P}, \beta_{\mathrm{O}}=\mathrm{n}_{\mathrm{O}}$, nombre de moles d'oxydant par unité de masse, $\beta_{\mathrm{T}}=\mathrm{c}_{\mathrm{p}}\left(\mathrm{T}-\mathrm{T}^{0}\right) / \Delta \mathrm{h}$, enthalpie sensible réduite, $\mathrm{T}^{0}$ température de référence. Enfin, le fluide étant compressible, on a introduit la transformation de Howarth pour définir les variables d'espace $\xi=\underline{\int} \rho \mu \mathrm{dx}$ et $\zeta=\underline{\int} \rho$ dy. La quantité -f (0) est une fonction bien déterminée de B. On obtient aisément les évolutions de concentrations et de la température dans le plan. La figure 12 montre la configuration du problème et la courbe $-\mathrm{f}(0)$ en fonction de $\mathrm{B}$.

Pour être applicable, la théorie d'Emmons requiert l'utilisation des hypothèses de Shvab-Zel'dovich. Plusieurs facteurs la mettent en défaut dont :

- La pesanteur qui fait monter les gaz chauds (Fig. 11a et b),

- La présence de suies qui induit des pertes radiatives,

- La perte d'enthalpie à la paroi thermo conductive et en cours de pyrolyse.

Il a été proposé de modifier le paramètre de Spalding $\mathrm{B}$ et de le remplacer par le paramètre de Torero $\mathrm{B}_{\mathrm{T}}$ qui tient compte de deux derniers effets mais pas de la force d'Archimède (réf. vii). L'usage de ce nouveau paramètre s'est révélé fructueux dans les expériences en micropesanteur.

Les suies se forment au cours de la combustion d'espèces carbonées. Leur nucléation et leur croissance ainsi que leur interaction avec l'écoulement réactif ont été étudiées par de nombreux auteurs. La prise en compte des suies a nécessité la mise au point de mécanismes adaptés et de tenir compte de l'interaction avec l'écoulement. Dorey ${ }^{x i}$ a fait une revue complète de ce problème complexe. Il y a quatre processus distincts: 1) nucléation, 2) croissance en surface, 3) oxydation et 4) coagulation. Il faut ensuite prendre en compte l'interaction de ces mécanismes de formation des suies avec l'écoulement en combustion et les transferts radiatifs. 


\section{Poudres}

\subsection{Thermites}

Un exemple de milieu solide donnant lieu, en l'absence de gaz, à un phénomène réactif très exothermique est constitué par les thermites, mélanges poudreux d'aluminium métallique et d'oxyde d'un autre métal.
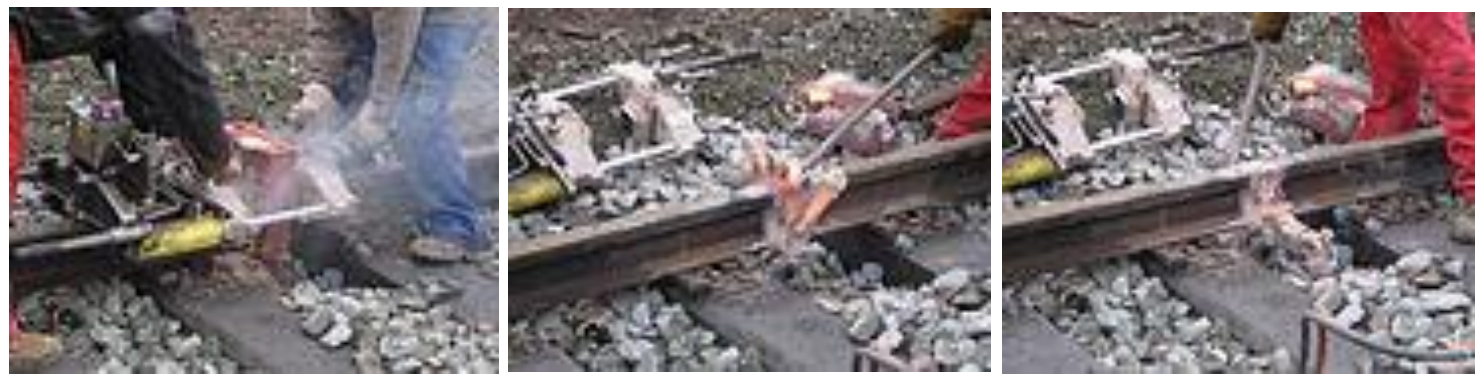

Figure 13. Utilisation d'une thermite pour le soudage de rails. Les températures atteintes lors des réactions chimiques en phase solide de l'aluminium avec des oxydes de fer sont assez élevées pour permettre le soudage. https://commons.wikimedia.org/wiki/File:Thermite_welding_16.JPG

On les utilise par exemple depuis longtemps pour souder des rails de chemin de fer ${ }^{11}$ (figure 13). Les températures atteintes lors des réactions chimiques en phase solide de l'aluminium avec des oxydes de fer sont assez élevées pour permettre le soudage, comme dans les trois exemples suivants : $\mathrm{Fe}_{3} \mathrm{O}_{4}$ et $\mathrm{Al}\left(3088^{\circ} \mathrm{C}\right)$, $\mathrm{FeO}$ et $\mathrm{Al}\left(2500^{\circ} \mathrm{C}\right), \mathrm{Fe}_{2} \mathrm{O}_{3}$ et $2 \mathrm{Al}\left(2960^{\circ} \mathrm{C}\right)$.

\subsection{Fusées à poudre}

Un exemple dérivé est constitué par les fusées à poudre ${ }^{12}$ (figure 14). Le pain de poudre disposé dans le corps de la fusée est creux. Lorsqu'il est allumé, il libère des espèces gazeuses et des gouttes liquides (d'aluminium pour les poudres contenant ce métal ${ }^{\mathrm{xii}}$ ) sous l'effet de la chaleur. La combustion se manifeste donc dans le jet fluide hétérogène. Les températures atteintes sont très élevées de même que la poussée résultante.

L'un des problèmes est l'accumulation de l'alumine solide $\mathrm{Al}_{2} \mathrm{O}_{3}$ - produit de réaction - dans le moteur en amont de la tuyère de sortie. Les recherches sur les modes de combustion des poudres sont toujours d'actualité.

Prenons le cas du $1^{\text {er }}$ étage de la fusée euro-italienne Véga issu de la technologie de Ariane5. Il fonctionnera au butalane 68-20 associant $12 \%$ de liant à base de polybutadiène (buta), $68 \%$ de perchlorate d'ammonium $\mathrm{NH}_{4} \mathrm{ClO}_{4}$ (1) comme oxydant, à $20 \%$ d'aluminium (ane) comme combustible.

11 Contributeurs de Wikilivres. Soudage/Soudage par aluminothermie [Internet]. 7 février 2015 , 08 :41 UTC, https://commons.wikimedia.org/wiki/File:Thermite welding 16.JPG 


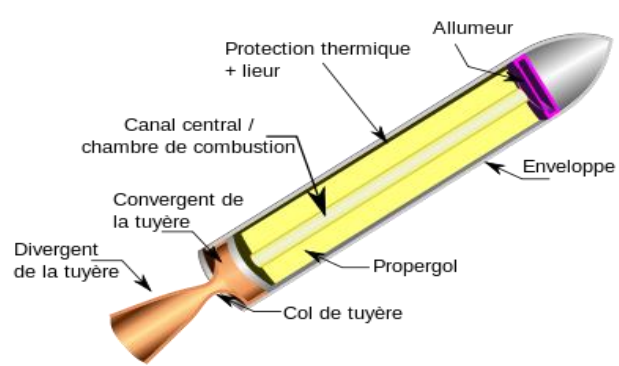

a)

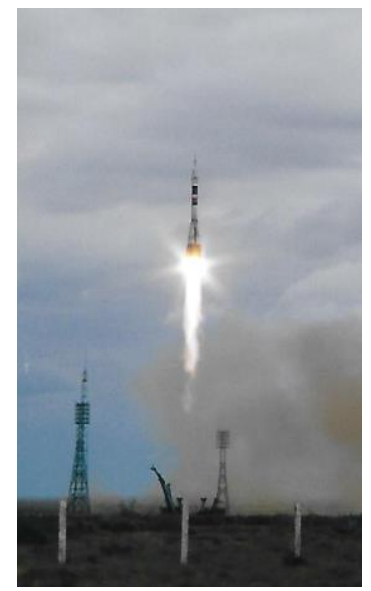

b)

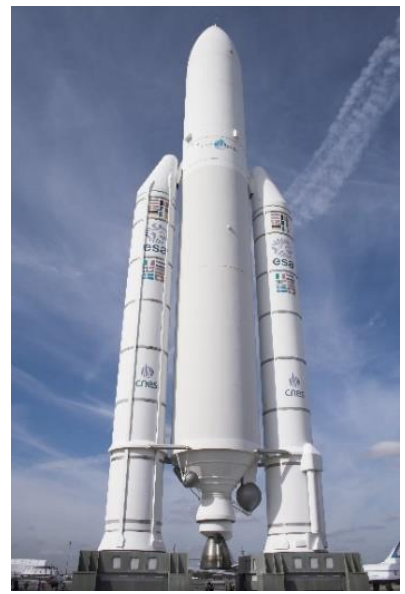

c)

Figure 14. a) Schéma d'un propulseur à propergol solide.

https://fr.wikipedia.org/wiki/Fichier:SolidRocketMotor-fr.svg, b) Lancement de la fusée Soyouz emportant des cosmonautes vers l'ISS le 21/10/2001, site de Baïkhonour13, c) La fusée Européenne Ariane. https://fr.wikipedia.org/wiki/Fichier:Ariane_5_Le_Bourget_FRA_001.jpg14.

Le processus de combustion du butadiène@ est complexe et comprend des réactions en phase gazeuse et une cinétique de surface. La température de flamme typique pour un propergol aluminisé (type Butalane $\left.{ }^{\circledR}\right)$ est de $3500 \mathrm{~K}$ ( $2300 \mathrm{~K}$ sans aluminium). La combustion des particules permet donc un apport d'énergie considérable. L'aluminium brûle à quelques $\mathrm{mm}$ de la paroi solide, dans la phase gazeuse et change peu la combustion du propergol (la flamme se situe à environ $100 \mu \mathrm{m}$ de la surface). On retrouve, à cette température, dans la phase gazeuse les produits finaux stables de décomposition du propergol à savoir : $\mathrm{N}_{2}, \mathrm{H}_{2} \mathrm{O}, \mathrm{CO}, \mathrm{CO}_{2}, \mathrm{HCl}$. Les particules micrométriques dont la surface est recouverte d'alumine (la couche d'alumine a une épaisseur d'environ $50 \AA$ ) quittent la surface du propergol et s'agglomèrent en partie en proche surface jusqu'à atteindre des diamètres d'environ 100 $\mu \mathrm{m} .70 \%$ d'entre elles se retrouvent directement dans l'écoulement de la chambre du propulseur. Dans un second temps cet agglomérat brûle, libérant des fumées d'alumine de petite taille (environ $1 \mu \mathrm{m}$ ). La combustion des agglomérats d'aluminium est généralement complète à condition que le temps de séjour dans le moteur soit suffisant. Cette combustion complète des agglomérats laisse des résidus d'alumine de taille de l'ordre de $10 \mu \mathrm{m}$.

Le schéma de la figure 14 vaut pour la combustion de chaque goutte d'aluminium liquide.

La description ébauchée précédemment exclue tout modèle réactif trop simplifié. Si l'on s'intéresse par exemple à la formation de l'alumine, on doit tenir compte non seulement des réactions en phase gazeuse, mais aussi des adsorptions-désorptions et chimie-sorptions qui mettent en jeu beaucoup d'autres espèces intermédiaires présentes aux hautes températures comme on le constate sur la figure 15.

Le seul mécanisme hétérogène peut compter 57 réactions élémentaires entre 58 espèces dont 2 à espèces de surface.

\footnotetext{
13 Depuis le retrait du service de la navette spatiale Etatsunienne en juillet 2011, le vaisseau Soyouz est le seul véhicule capable d'envoyer des humains dans l'espace (avec les fusées chinoises). Les divers étages sont équipés de moteurs à propergols liquides.

${ }^{14}$ Les lanceurs Ariane 5 ont d'abord une partie basse qui comprend d'une part un corps central équipé d'un moteur cryotechnique Vulcain, et d'autre part deux étages d'accélération à poudre servant à fournir l'essentiel de la poussée lors du décollage 


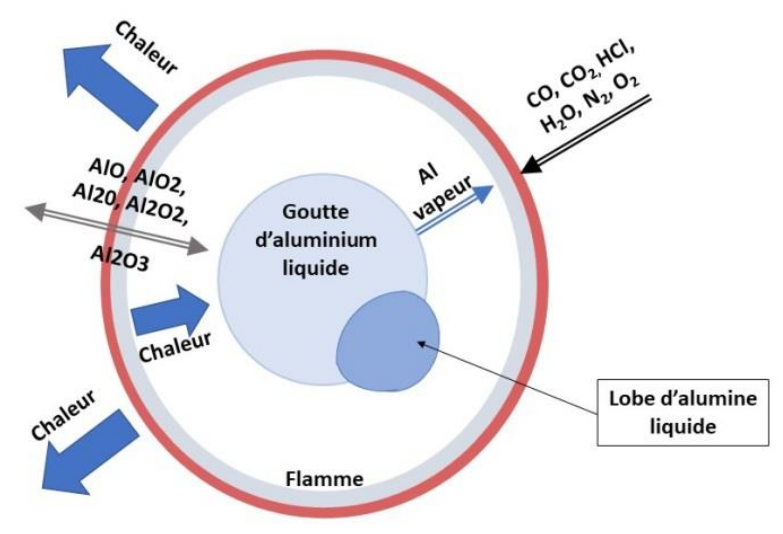

Figure 15. Schéma de la combustion d'une particule d'aluminium en ambiance propergol. Accord de l'auteur Glorian (réf. xii).

La formation de $\mathrm{Al}_{2} \mathrm{O}_{3}$, en présence d'air, l'indice ${ }_{\mathrm{L}}$ signifiant "liquide", fait intervenir les 5 réactions :

$$
2 \mathrm{Al}+3 / 2 \mathrm{O}_{2}-->\mathrm{Al}_{2} \mathrm{O}_{3 \mathrm{~L}} ; 2 \mathrm{AlO}+1 / 2 \mathrm{O}_{2}-->\mathrm{Al}_{2} \mathrm{O}_{3 \mathrm{~L}}
$$

$$
\mathrm{AlO}_{2}+\mathrm{AlO}-->\mathrm{Al}_{2} \mathrm{O}_{\mathrm{L}} ; \mathrm{Al}_{2} \mathrm{O}+\mathrm{O}_{2}-->\mathrm{Al}_{2} \mathrm{O}_{3 \mathrm{~L}} ; \mathrm{Al}_{2} \mathrm{O}_{2}+1 / 2 \mathrm{O}_{2}-->\mathrm{Al}_{2} \mathrm{O}_{3 \mathrm{~L}}
$$

Si l'on tient compte des espèces générées par le propergol, il y a aussi :

$$
\mathrm{Al}_{2} \mathrm{O}+2 \mathrm{CO}_{2}=\mathrm{Al}_{2} \mathrm{O}_{3}+2 \mathrm{CO} ; 2 \mathrm{AlO}+\mathrm{CO}_{2}=\mathrm{Al}_{2} \mathrm{O}_{3}+\mathrm{CO}
$$

$$
\mathrm{Al}_{2} \mathrm{O}+2 \mathrm{H}_{2} \mathrm{O}=\mathrm{Al}_{2} \mathrm{O}_{3}+2 \mathrm{H}_{2} ; 2 \mathrm{AlO}+\mathrm{H}_{2} \mathrm{O}=\mathrm{Al}_{2} \mathrm{O}_{3}+\mathrm{H}_{2} \text {. }
$$

Seul le calcul numérique peut venir à bout d'un tel problème qui contient également ce qui est relatif aux divers transports telle la convection, les diffusions, le rayonnement.

\subsection{Combustion en spirale}

Donnons un dernier exemple de combustion de solides qui peut se révéler très utile pour la récolte de données de cinétique chimique.

Comme signalé en section 4.2 à propos des flammes de prémélange, des mesures réalisées en combustion des solides sont utiles également pour déterminer des quantités de cinétique chimique. Holt et al. (https://doi.org/10.1016/0025-5416(85)90244-7) ont pu par exemple utiliser la combustion de TiB2. D'autres réactions de synthèse ont été étudiées concernant des produits utiles pour l'électronique et les batteries d'accumulateurs. Il nous a semblé intéressant de montrer ici un mode de propagation en surface de ces combustions faisant l'objet de recherches récentes. Certaines études portent en effet sur la propagation en spirale de sites de nucléation de combustion. Il s'agit de la combustion en surface de cylindres de poudre métallique. En particulier A.I. Kirdyashkin et al ont étudié expérimentalement les ondes de combustion de poudre ${ }^{\text {xii }}$ sous faisceau laser ${ }^{\text {xiv }}$. 


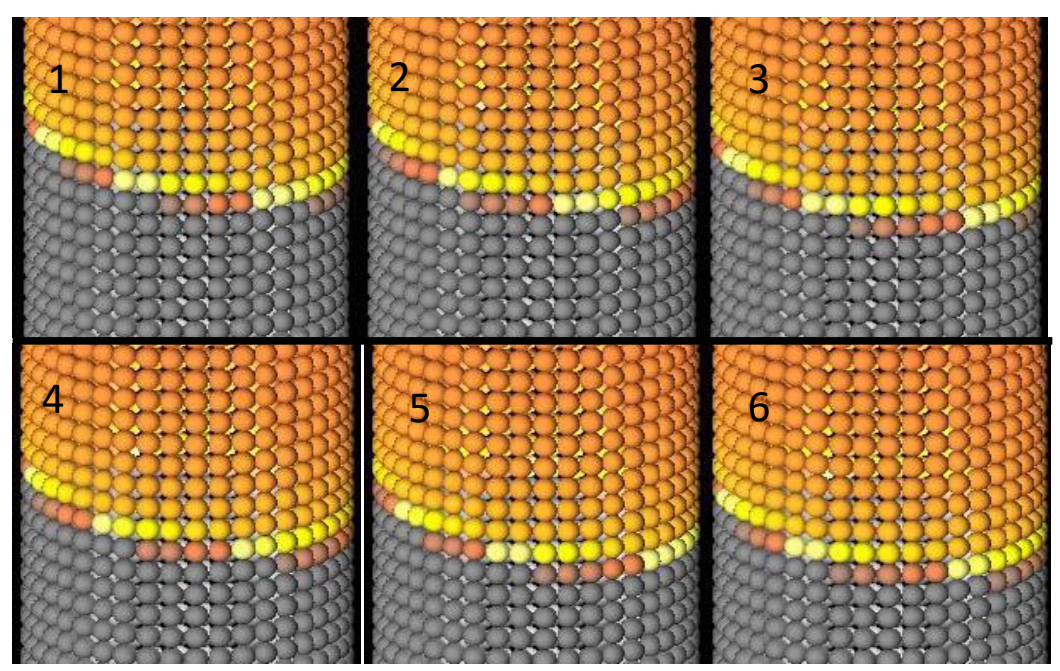

Figure 16. Propagation d'une onde de combustion discrète en mode spin multicouche (les particules claires sont enflammées ; les particules foncées sont non-ignifugées). La combustion a lieu vers le bas ; the spin se meut le long de la surface de droite à gauche [réf. 23] APS. Licence $n^{\circ} R N P$ _18_AUG_007366.

Plus récemment Klimenok et Rashkovskiy ${ }^{\mathrm{xv}}$ ont publié un modèle discret de « spin-combustion » de mélange de poudres en l'absence de gaz. La figure 16 montre la progression de la combustion en surface. Bharath et $\mathrm{al}^{\mathrm{xvi}}$ estiment que le processus peut parfois être de nature purement thermique et relié à l'hétérogénéité des systèmes dispersés et à l'existence d'une température seuil.

\section{Sprays et feux d'artifice}

Le caractère hétérogène se présente également avec les propergols liquides. On assiste alors le plus souvent à la combustion de sprays (figure 17).

La connaissance de la distribution en taille des sprays est essentielle pour l'obtention d'une combustion optimale dans les moteurs afin de récupérer au mieux l'énergie et de diminuer la quantité de produits polluants ${ }^{15}$.

Cela vaut pour les moteurs terrestres comme pour les avions et les fusées. Dans le cas du moteur cryotechnique de la fusée Ariane, le spray est composé de gouttes d'oxygène liquide dans de l'hydrogène gazeux et les conditions de fonctionnement sont de plus supercritiques ${ }^{16}$.

Des instabilités de combustion peuvent se présenter. Ce problème est crucial car celles-ci peuvent causer des dommages allant jusqu'à la destruction. Certaines instabilités sont dues à l'amplification des vibrations en provenance du moteur sous l'effet de l'évaporation des gouttelettes issues des injecteurs.

\footnotetext{
${ }^{15} \mathrm{https}: / /$ www.encyclopedie-environnement.org/air/particules-polluantes-air/

${ }_{16} \mathrm{Au}$-dessus du point critique liquide-vapeur, il n'y a plus de différence entre liquide et vapeur, il n'y a plus qu'un seul fluide dit supercritique. L'interface entre l'oxygène liquide et l'hydrogène gazeux ne présente plus de tension superficielle comme c'était le cas en régime subcritique. Le point critique est un point singulier. En son voisinage le coefficient de conduction thermique tend vers zéro. Il apparait un nouveau mode de transfert thermique appelé "effet piston". 


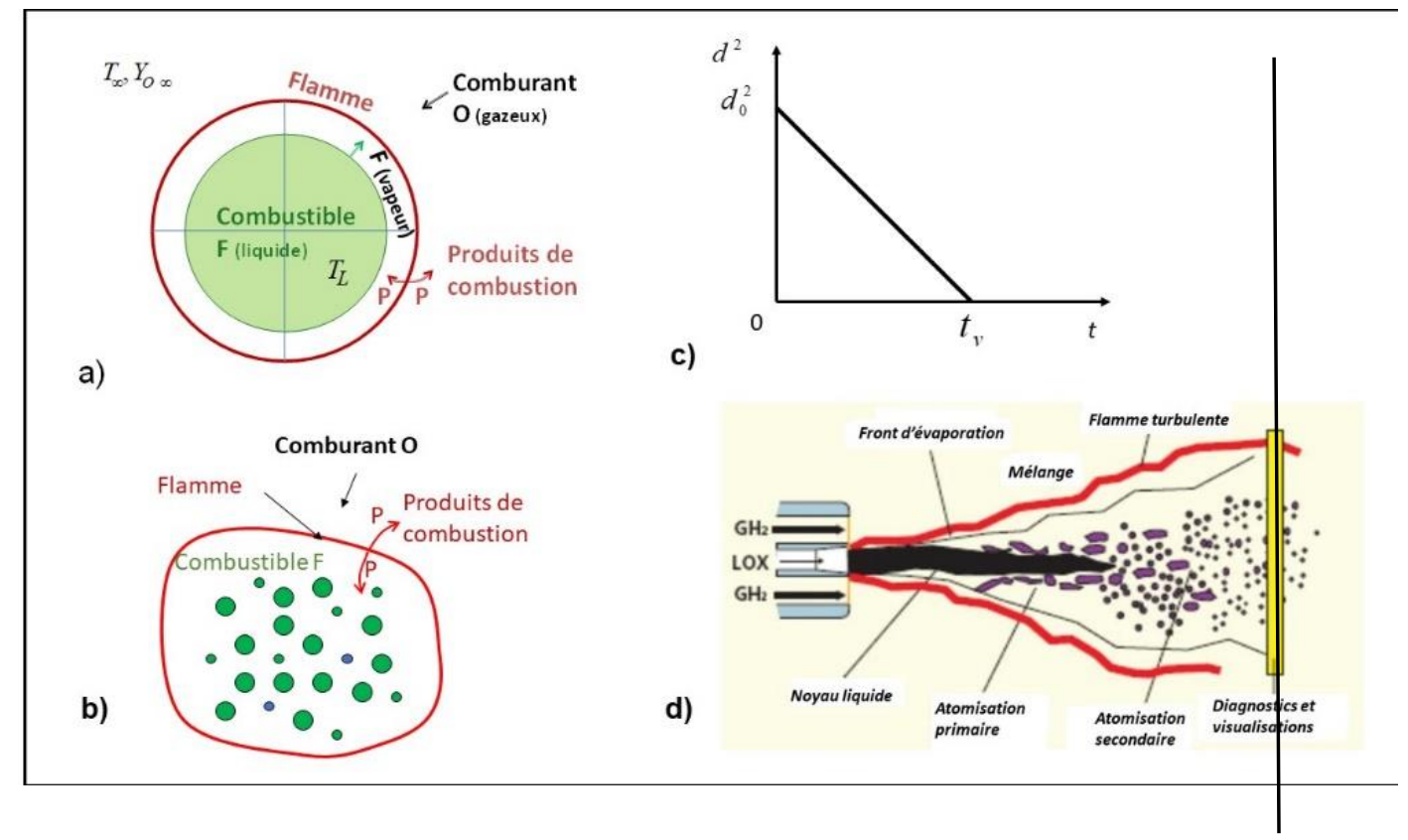

Figure 17. Combustion de sprays : a) Goutte individuelle en combustion avec flamme de diffusion sphérique17 ; b) Paquet de gouttes en combustion, chaque goutte étant en évaporation ; c) Dans les deux cas l'évolution du diamètre $d$ de goutte suit assez bien une loi en $d 2=d 02(1-t / t v)$; tv durée de vie ; d) Le phénomène physique induit dans la flamme issue d'un injecteur coaxial cryotechnique (d'après le site web de I'ONERA).

Une autre situation hétérogène se manifeste avec les feux d'artifice à main. Inoue et al. ont étudié le problème de la fragmentation en cascade ${ }^{\mathrm{xvii}}$. A ce titre ils ont examiné en particulier l'évolution d'une gouttelette perturbée par l'éclatement d'une cavité gazeuse interne. Ils montrent ainsi que la goutte peut osciller autour de sa forme sphérö̈dale initiale lorsque l'énergie mise en jeu est plus petite qu'une certaine valeur critique. Lorsque l'énergie mise en jeu est suffisamment élevée, la goutte peut aussi se déformer en un ligament assez allongé pour se fragmenter. La figure 18 montre la combustion d'un cierge magique.

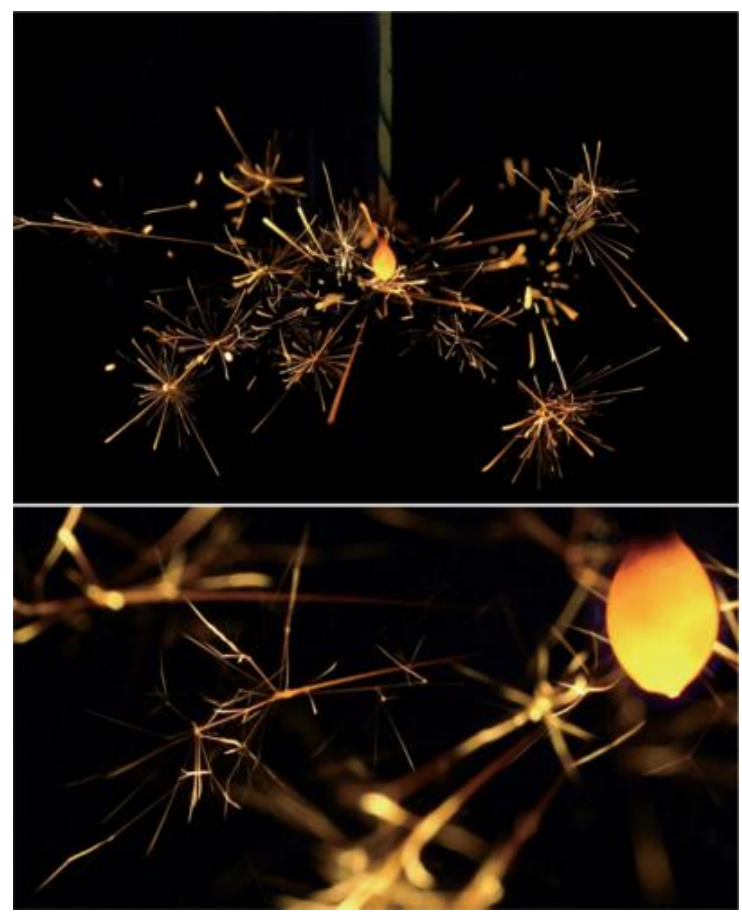

Figure 18. Traces des chemins de gouttelettes illustrant le processus continu de fragmentation en cascade dans un feu d'artifice traditionnel. En haut : temps d'exposition 1/60 s, le cordon de papier de $2 \mathrm{~mm}$ de dia-

\footnotetext{
${ }^{17}$ Le spray en combustion est pris comme exemple d'écoulement hétérogène, mais à l'échelle de la goutte, il s'agit bien d'une flamme de diffusion en milieu homogène. 
mètre retient la goutte mère. En bas: Un temps d'exposition plus long de 1/25 s révèle la cascade de ruptures successives avec jusqu'à quatre générations visibles sur l'arborescence au foyer. [réf. 27], APS. Numéro de Licence RNP_18_AUG_007267.

Les flammes, tout comme les feux d'artifice, émettent de la lumière car elles contiennent des corps à haute température. Nous n'exposerons pas les mécanismes radiatifs qui en sont la cause dans ce court article sur la combustion.

\section{Messages à retenir}

- La combustion résulte de la libération d'énergie de « réactants » par des réactions chimiques donnant des « produits de réaction » dits brûlés.

- Elle peut se produire en milieu fluide ou solide, homogène ou hétérogène. Dans les mélanges gazeux elle donne lieu à la formation de flammes de différents types, qui se développent en écoulement laminaire ou turbulent. En ce qui concerne les solides, il s'agit généralement de poudres compactées.

- Son étude à des fins de recherche ou de prévision nécessite de faire les bilans des grandeurs physiques qui interviennent dans les mélanges : masse des espèces, quantités de mouvement, énergies. Elle implique la connaissance de la cinétique chimique et des lois de comportement des milieux. La résolution, généralement numérique, a donné lieu à la mise au point de nombreuses méthodes spécifiques dépassant le cadre de cette présentation.

- Il s'agit d'un sujet très vaste intervenant dans de nombreux domaines et qui a un historique abondant, non abordé ici. Ces quelques éléments de base sont fournis pour aider le lecteur à aborder certains sujets environnementaux faisant intervenir la combustion.

\section{En Savoir plus :}

Les revues Combustion and Flame, Combustion Science and Technology.

Proceedings of the Combustion Institute.

Sur la «spin combustion » dans les poudres ${ }^{\mathrm{xviii}, \mathrm{xix}, \mathrm{xx}, \mathrm{xxi}}$

Sur les phénomènes radiatifs ${ }^{\text {xxii,xxiii }}$

Sur le mélange en combustion ${ }^{\text {xxiv }}$

\section{Remerciements}

L'auteur tient à remercier Hayat pour sa patiente relecture des versions successives. Sa reconnaissance va également à Joël Sommeria pour son expertise et ses critiques constructives.

\section{Annexes}

Les deux annexes qui suivent complètent le corps de l'article. L'annexe 1 donne la base et le détail des calculs de la théorie de Rankine-Hugoniot des ondes de combustion. L'annexe 2 tente un historique de la science de la combustion. 


\section{La théorie de Rankine-Hugoniot}

Nous avons vu en section 2 qu'une flamme de diffusion se positionnait au contact entre une zone comburante et une zone de combustible. Elle est en quelque sorte un lieu géométrique de points à concentration stœchiométrique.

La position d'une flamme de prémélange (section 3) n'est au contraire pas déterminée a priori. Le phénomène de combustion qui se développe dans un prémélange homogène est une onde de discontinuité résultant des interactions mettant en jeu convection, diffusion de la chaleur et des espèces et réaction chimique avec son énergie d'activation et son énergie libérée. La théorie de Rankine-Hugoniot s'intéresse à ce type d'onde de discontinuité dans le cas stationnaire. Pour établir les caractéristiques d'une telle onde il faut d'abord établir les bilans des grandeurs physiques à sa traversée en la traitant comme une discontinuité de l'écoulement fluide.

\section{A1. Les bilans}

Rappelons d'abord ce que l'on entend par bilan d'une grandeur extensive $\mathrm{F}$ dans un écoulement fluide continu. Nous aborderons ensuite le bilan de F an niveau d'une discontinuité. A ce niveau de généralité nous éviterons autant que possible les grands développements mathématiques, pourtant utiles ensuite si l'on veut passer aux applications.

\section{A1.2. Bilan continu}

Le bilan d'une quantité $\mathrm{F}$ dans un volume $(V)$ arbitraire de milieu continu s'écrit formellement :

Variation de $\mathrm{F}$ dans $(V)+$ Flux sortant de $\mathrm{F}$ à travers $(\partial V)=$ Production de $\mathrm{F}$ dans $(V)$.

Ce bilan se traduit mathématiquement par l'équation locale de bilan de la grandeur $\mathrm{F}$ dans un volume de contrôle (Figure 19) connexe arbitraire $V$ limité par sa frontière $(\partial V)$.

S'il existe un référentiel dans lequel l'état du système est invariable dans le temps, l'évolution y est stationnaire. En choisissant alors la frontière $(\partial V)$ fixe, le premier terme de l'équation du bilan disparait.

Si la grandeur F est conservative, la production est nulle par définition et c'est le second membre de l'équation du bilan qui disparait.

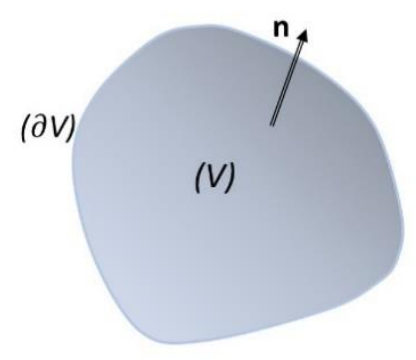

Figure 19. Volume de contrôle $(V)$, la normale $n$ à sa frontière $(\partial V)$. 
Pour une évolution stationnaire, l'équation de bilan non conservative a donc la forme

Flux sortant de $\mathrm{F}$ à travers $(\partial V)=$ Production de $\mathrm{F}$ dans $(V)$

Elle s'écrit mathématiquement $\int_{(\partial V)} \mathbf{J}_{\mathrm{F}} \cdot \mathbf{n} \mathrm{d} S=\underline{\int}_{(V)} \dot{W}_{\mathrm{F}} d V$ pour un volume $(V)$ quelconque. On démontre que localement

$\nabla \cdot \mathbf{J}_{\mathrm{F}}=\dot{W}_{\mathrm{F}}$

\section{A1.2. Bilan discontinu}

L'équation générale du bilan de $\mathrm{F}$ à travers une surface discontinuité dans un écoulement peut s'écrire formellement de la même manière qu'en milieu continu, mais cette fois le volume $(V)$ est remplacé par une portion d'interface réelle $(S)$ (Figure 20) limitée par une courbe fermée $(C)$. Si la discontinuité est stationnaire, c'est qu'il y a un référentiel dans lequel elle est au repos et où le premier terme de l'équation de bilan - celui de la variation de $\mathrm{F}$ dans $(S)$ - s'annule.

En l'absence de flux à travers la ligne $(C)$, ce qui signifie en particulier que l'on néglige les tensions de surface, il reste la relation :

$$
\left(\mathbf{J}_{\mathrm{F} 2^{-}} \mathbf{J}_{\mathrm{F} 2}\right) \cdot \mathbf{N}=\dot{W}_{a \mathrm{~F}}
$$

qui veut dire que le saut du flux $\mathbf{J}_{\mathrm{F}}$ projeté sur la normale $\mathbf{N}$ à la traversée de $(S)$, est égal au taux de production de $\mathrm{F}$ par unité d'aire (indice $a$ ). On écrit aussi $\mathrm{J}_{\mathrm{FN} 2}-\mathrm{J}_{\mathrm{FN} 1}=\dot{W}_{a \mathrm{~F}}$, avec l'indice $\mathrm{N}$ pour la projection normale à $(S)$.
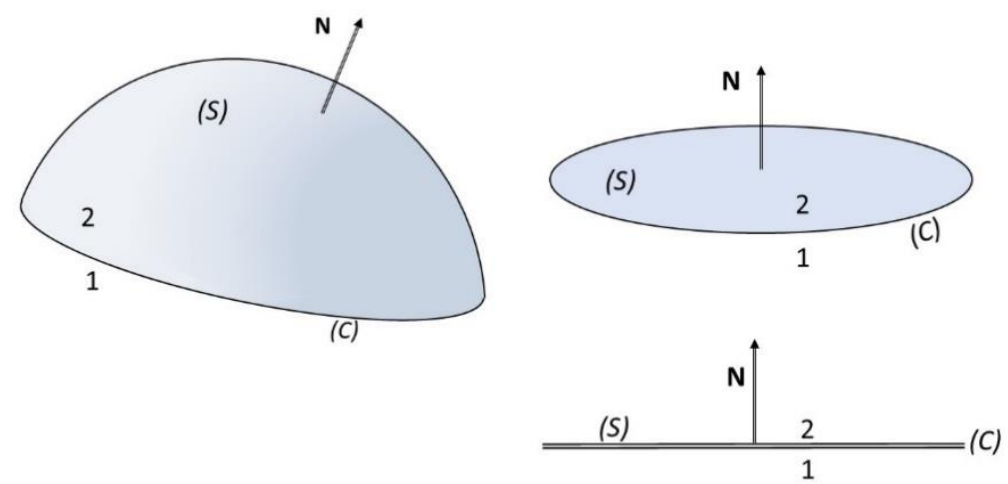

Figure 20. Interface de discontinuité. La normale $\mathbf{N}$ à la discontinuité est orientée dans le sens de la vitesse relative du fluide qui la traverse. A gauche : interface incurvée; à droite en haut : interface plane, en bas vue de profil

L'équation de saut ainsi obtenue s'applique aux ondes de choc stationnaires en fluide simple compressible :

- Pour les quantités conservatives : masse, quantité de mouvement, énergie totale on a $\dot{W}_{a \mathrm{~F}}=0$ et le bilan de F s'écrit localement

$$
\mathrm{J}_{\mathrm{FN} 2}-\mathrm{J}_{\mathrm{FN} 1}=0
$$

- Pour les quantités non conservatives telle l'entropie $S$, il apparaît un second membre qui est le taux de production de la grandeur $\mathrm{F}$ à l'intérieur de l'interface

$$
\mathrm{J}_{\mathrm{FN} 2}-\mathrm{J}_{\mathrm{FN} 1}=\dot{W}_{a \mathrm{~F}}
$$


Ces équations de bilan s'appliquent aux corps purs aussi bien qu'aux mélanges.

Dans un gaz non réactif les seules ondes de discontinuité sont des ondes de compression appelées ondes de choc qui ont la propriété d'avoir une vitesse de propagation supersonique par rapport au gaz amont. Les ondes de détente, telles que les ondes centrées, existent mais elles sont des phénomènes continus.

Dans un mélange gazeux réactif, ce n'est pas la même chose, car nous verrons (en A3) qu'il peut se développer, pour un même mélange, deux types d'ondes de discontinuité.

\section{A2. L'onde de choc droite stationnaire}

Parmi les exemples les plus simples de discontinuités, il y a les ondes de choc droites (ou planes) ${ }^{\mathrm{xxv}}$. Ce problème est déjà abondamment traité. On connait bien toutes les formules des ondes de choc droites stationnaires dans un gaz parfait.

En plus des ondes de choc droites, il existe des ondes de choc obliques et des chocs courbes. Les figures 21 à 23 illustrent les ondes de choc droites stationnaires.

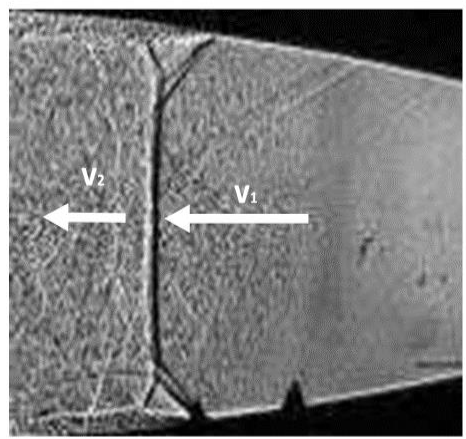

a)

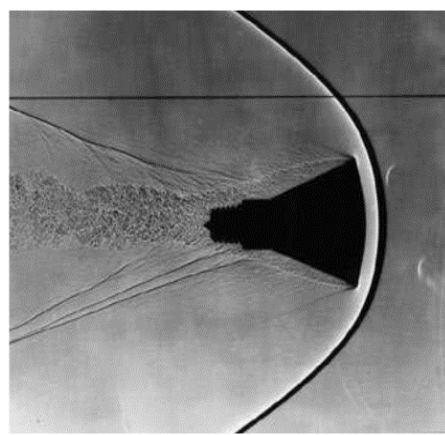

b)

Figure 21. Visualisations de chocs. a) Choc droit dans le divergent d'une tuyère de Laval, b) Choc courbe à l'avant d'un corps de rentrée, mais assimilable à un choc droit au voisinage de l'axe de symétrie

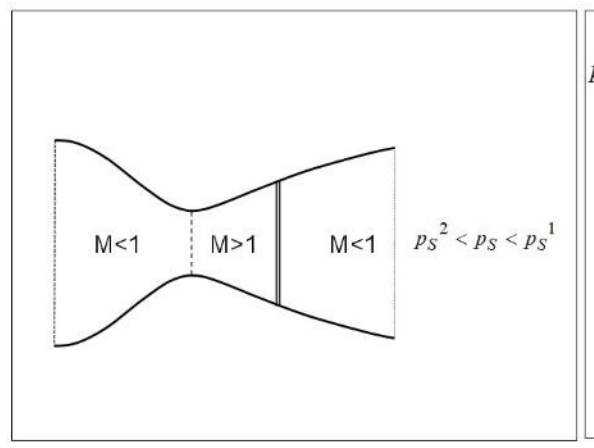

a)

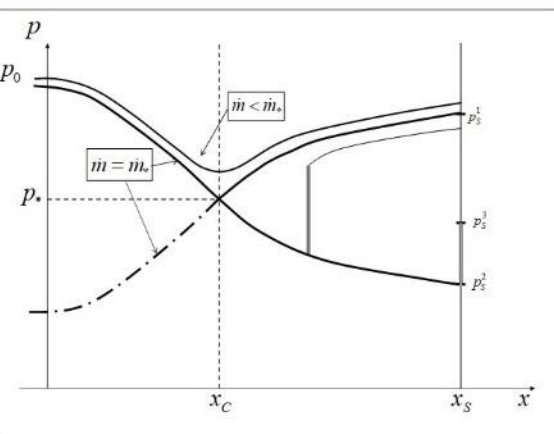

b)

Figure 22. a) Schéma d'une tuyère de Laval avec choc dans le divergent b) Solutions d'écoulement dans une tuyère de Laval. Le profil de tuyère est donné par la fonction $A=A(x)$. Les courbes correspondant au débit masse critique $\dot{m}=\dot{m}$ * sont des asymptotes pour les écoulements internes continus, la partie en trait mixte est fictive. 
Les équations de bilan d'une onde de choc droite stationnaire sont les suivantes :

\begin{tabular}{|c|}
\hline$\left(\rho_{2} u_{2}-\rho_{1} u_{1}\right)=\left(\dot{m}_{2}-\dot{m}_{1}\right)=0$ (masse) \\
\hline$\dot{m}\left(u_{2}-u_{1}\right)+p_{2}-p_{1}=0$ (quantité de mouvement) \\
\hline$\dot{m}\left(h_{2}-h_{1}+u_{2}^{2} / 2-u_{1}^{2} / 2\right)=0$ (énergie) \\
\hline$\dot{m}\left(s_{2}-s_{1}\right) \geq 0$ (entropie) \\
\hline
\end{tabular}

Dans ces équations $\dot{m}$ est le débit masse unitaire (c'est-à-dire par unité d'aire) ; $\rho_{\mathrm{i}}$ est la masse volumique $\mathrm{i}=1,2 ; \mathrm{p}_{\mathrm{i}}$ est la pression, $h_{\mathrm{i}}$ l'enthalpie massique, $u_{\mathrm{i}}$ la vitesse relative par rapport à l'onde, $s_{\mathrm{i}}$ est l'entropie massique.

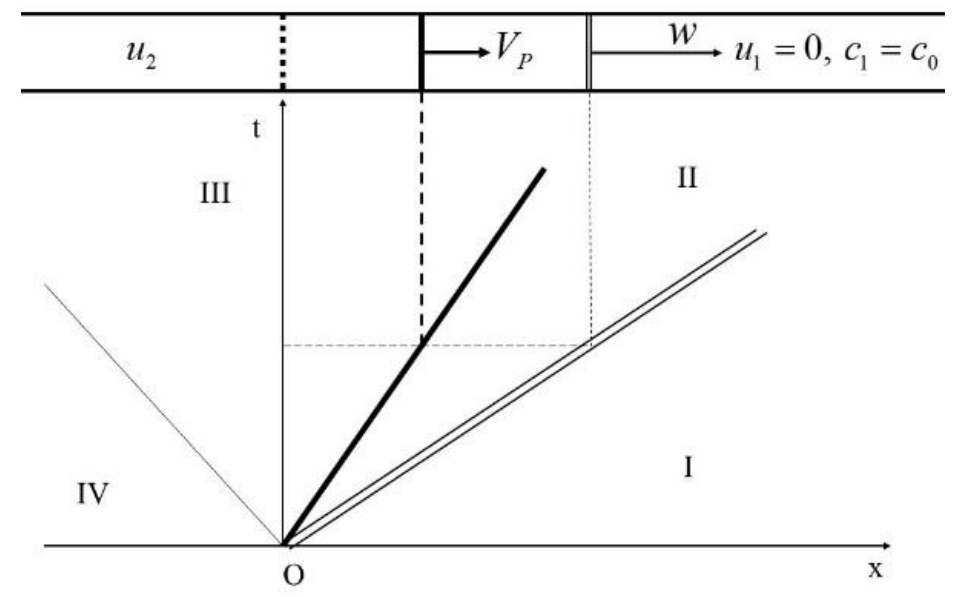

Figure 23. Mouvement de part et d'autre d'un piston de vitesse uniforme $V_{P}$. La trajectoire du piston est en traits pleins épais, celle de l'onde de choc de vitesse $w$ en trait double. A l'avant du choc, dans la région I, le gaz est au repos $u_{1}=0$, la célérité $d u$ son est $c_{0}$.

On pose $p=p_{2} / p_{1}, \vartheta=\rho_{1} / \rho_{2}, \gamma=c_{\mathrm{p}} / c_{\mathrm{v}}, M_{1}=u_{1} / c_{1}$ est le nombre de Mach de l'écoulement amont, $c_{1}=\gamma p_{1} /$ $\rho_{1}$ est la célérité du son, $c_{\mathrm{p}}$ et $c_{\mathrm{v}}$ sont les chaleurs massiques du gaz.

La droite de débit a pour équation : $(p-1) /(\vartheta-1)=-\gamma M_{1}^{2}$ et le taux de production d'entropie $\dot{W}_{\mathrm{Sa}}$ est toujours positif ou nul d'après le second principe de la thermodynamique. Compte tenu des lois d'état des gaz parfaits : $\dot{m}\left(s_{2}-s_{1}\right)=\dot{m} c_{v} \ln (\mathrm{p} \vartheta \gamma$ ) en désignant par $p$ et $\vartheta$ la pression et le volume massique rapportés à leurs valeurs amont.

La figure A6 24a indique la détermination graphique du point de fonctionnement de l'onde de choc droite dans le plan de Clapeyron et la figure 6 24b donne le saut d'entropie le long de l'adiabatique d'Hugoniot.

Une onde de choc correspond à une zone d'écoulement de très faible épaisseur, inférieure à $1 \mathrm{~mm}$, qui fait la transition entre l'amont où la vitesse est supersonique et l'aval où elle est subsonique. $\mathrm{A}$ la traversée du choc, la pression augmente très fortement. Ainsi, les particules du fluide lors de leur traversée de l'onde de choc subissent une décélération extrêmement forte qui peut atteindre $10^{9}$ à 
$10^{10} \mathrm{~m} / \mathrm{s}^{2}$, soit environ un milliard de $g$. C'est cet impact violent, produisant un véritable choc sur les particules, qui est à l'origine du nom donné à cette zone de transition ${ }^{\text {xxvi }}$. L'onde de choc est toujours une discontinuité de compression.

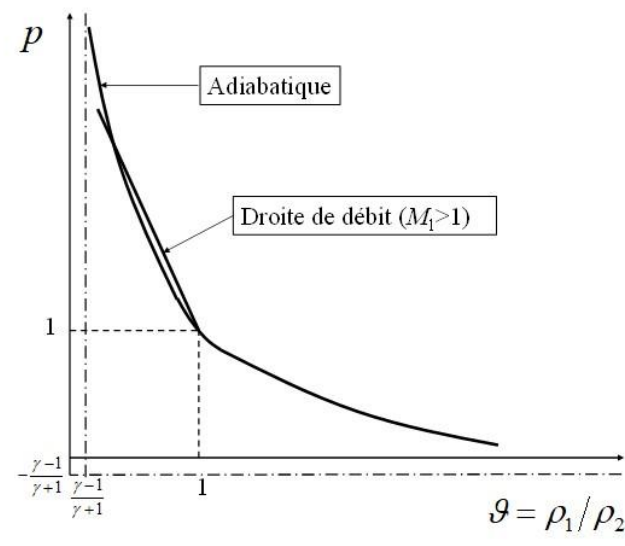

a)

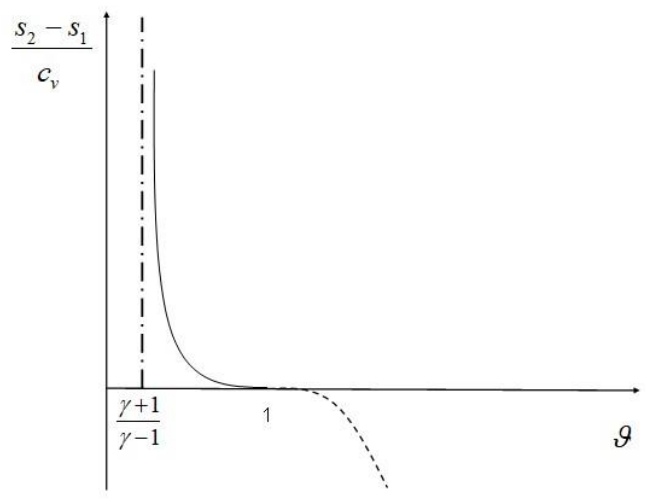

b)

Figure 24. L'adiabatique d'Hugoniot dans les plans a) $\vartheta$, $p$ et b) $\vartheta$, s. Le nombre de Mach de l'écoulement amont par rapport à l'onde est : $M_{1}=U_{1} / c_{1}$. II n'y a de choc que pour $M_{1}>1$.

\section{A3. Ondes de combustion stationnaires}

\section{A3.1. Les diagrammes}

Les équations de Rankine-Hugoniot caractérisent une onde de discontinuité plane en présence d'une réaction chimique de combustion. Les équations de base sont analogues à celles de l'onde de choc. Ainsi les équations à la discontinuité s'écrivent, successivement pour la masse, la quantité de mouvement et l'énergie, comme pour l'onde de choc :

$\left(\rho_{2} u_{2^{-}} \rho_{1} u_{1}\right)=\left(\dot{m}_{2^{-}} \dot{m}_{1}\right)=0$ (masse), $\dot{m}\left(u_{2}-u_{1}\right)+p_{2}-p_{1}=0$ (quantité de mouvement),

$\dot{m}\left(h_{2}-h_{1}+u_{2}^{2} / 2-u_{1}^{2} / 2\right)=0$ (énergie),

mais il faut leur ajouter les équations d'état du mélange qui sont en partie différentes de celles de l'onde de choc.

En simplifiant et en prenant une chaleur spécifique $c_{\mathrm{p}}$ moyenne on obtient :

$h_{2}-h_{1}=-\Delta h+c_{\mathrm{p}}\left(T_{2}-T_{1}\right)$

alors qu'à la traversée d'un choc sans combustion on a : $h_{2^{-}} h_{1}=c_{\mathrm{p}}\left(T_{2^{-}} T_{1}\right)$. 


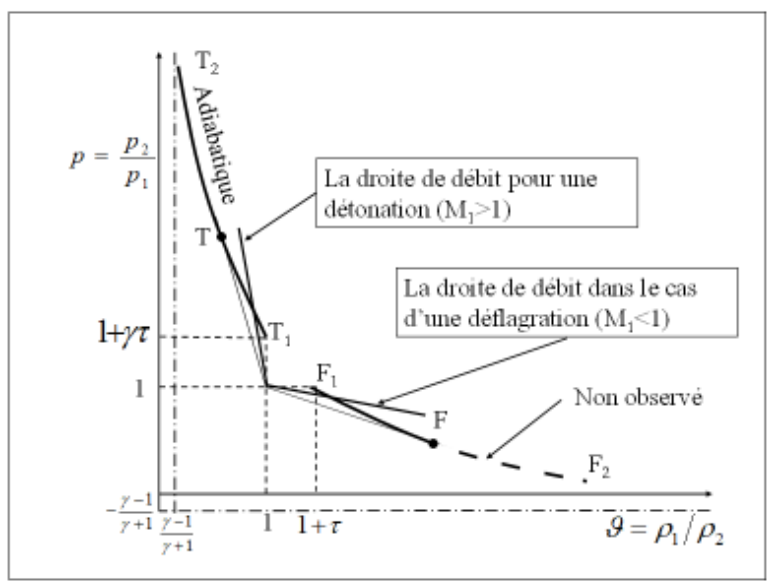

a)

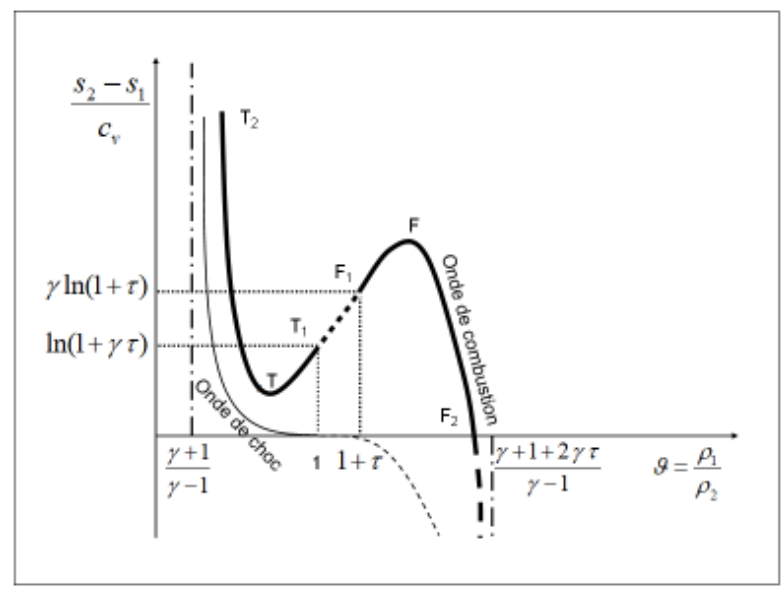

b)

Figure 25. a) La droite de débit (dans deux cas) $M_{1}=u_{1} / c_{1}$ désignant le nombre de Mach de l'onde par rapport aux gaz frais, et l'adiabatique d'Hugoniot. Les points de fonctionnement se trouvent à l'intersection de ces deux courbes b) Taux de production d'entropie, à la traversée de l'onde de combustion, le long de l'adiabatique d'Hugoniot. Notations : coefficient isentropique $\gamma=c_{p} / c_{v}$, saut d'entropie $\left(s_{2}-s_{1}\right)$, masse volumique $\rho$, énergie réduite mise en jeu $\tau=\Delta h / c_{p} T_{0}$.

En éliminant $u_{1}$ et $u_{2}$ entre l'équation de la masse et celle de la quantité de mouvement et, en posant $\vartheta=\rho_{1} / \rho_{2}, p=p_{2} / p_{1}$ on obtient, après simplification, les deux équations suivantes dites « relations de Rankine-Hugoniot » où $M_{1}=u_{1} / c_{1}$, rapport de la vitesse à la célérité du son dans les gaz frais, est le nombre de Mach amont

$(p-1) /(\vartheta-1)=-\gamma M_{1}^{2}($ droite de débit $)$
$p=[(1-\gamma) \vartheta+1+\gamma+2 \gamma \tau] /[(1+\gamma) \vartheta+1-\gamma]$ (adiabatique d'Hugoniot)

décrivant respectivement la droite des débits et l'adiabatique d'Hugoniot. La donnée du nombre de Mach amont $\mathrm{M}_{1}$ permet de résoudre le système. Sur la figure 25, l'arc $F_{1} F F_{2}$ de l'adiabatique d'Hugoniot est dans la zone de variation d'entropie positive et rend possible une onde de discontinuité d'expansion. Le choc représenté en trait fin, ne présente pas une telle onde.

\section{A3.2. Les résultats}

Sur la figure 25a, les points de fonctionnement se trouvent à l'intersection de la droite de débit à $\mathrm{M}_{1}$ donné et de l'adiabatique d'Hugoniot, mais la figure $25 \mathrm{~b}$ indique les conditions d'existence des solutions, car la variation de l'entropie $\left(s_{2}-s_{1}\right)$ doit être obligatoirement positive. On note aussi la présence d'un minimum sur la courbe de variation de l'entropie. Ce minimum correspond à un point stable dans le plan de Clapeyron de la figure 25a.

Cette théorie simplifiée ${ }^{\mathrm{xxvii}}$ résumée en section 3.1 rend bien compte des différentes ondes de combustion planes stationnaires en régime laminaire, susceptibles de se présenter dans un tube rectiligne. Ce tube est rempli initialement d'un mélange de comburant et de combustible et ses parois sont adiabatiques (c'est-à-dire qu'elles ne permettent pas d'échange de chaleur avec l'extérieur). L'allumage à l'une des extrémités du tube permet d'abord la progression dans les gaz frais d'une onde lente dont la vitesse est de l'ordre du $\mathrm{m} / \mathrm{s}$ - dite de déflagration - qui n'est rien d'autre qu'une flamme 
plane de prémélange. Puis au cours du temps, cette onde s'accélère et devient une onde de détonation très rapide progressant à vitesse supersonique.

En résumé, l'onde de compression existe comme dans le cas non réactif, c'est une onde de choc boostée par l'énergie de la réaction chimique, donc plus intense et tout à fait supersonique (2000 à 3000 $\mathrm{m} / \mathrm{s}$ ), que l'on appelle détonation. Mais l'existence d'ondes de détente discontinues est rendue aussi possible par les lois de la thermodynamique. Ce sont les ondes de déflagration, qui sont nettement subsoniques.

Dans un système d'axes lié à l'onde le phénomène peut être schématisé comme sur la figure A8 :

- - en amont de l'onde ( $x<0$ ), le mélange de gaz frais se déplace à vitesse constante $\mathbf{v}_{1}$,

- - à la traversée de l'onde $(x=0)$ le fluide subit une discontinuité,

- - en aval de l'onde $(x>0)$ le mélange de gaz brûlés se déplace en mouvement uniforme à la vitesse $\mathbf{v}_{2}$.

Dans la zone de vitesse $\mathbf{v}_{1}$ les concentrations sont celles des gaz frais, la température est trop faible pour que les réactions chimiques aient lieu. Le mélange est figé. Dans la zone de vitesse $\mathbf{v}_{2}$ on a affaire à un mélange gazeux en équilibre chimique. L'enthalpie $h_{2}$ se rapporte donc à un gaz d'une autre nature que le gaz (1). La différence des enthalpies correspond à l'énergie mise en jeu par la réaction à laquelle s'ajoute l'enthalpie de chauffage du gaz entre $T_{1}$ et $T_{2}$.

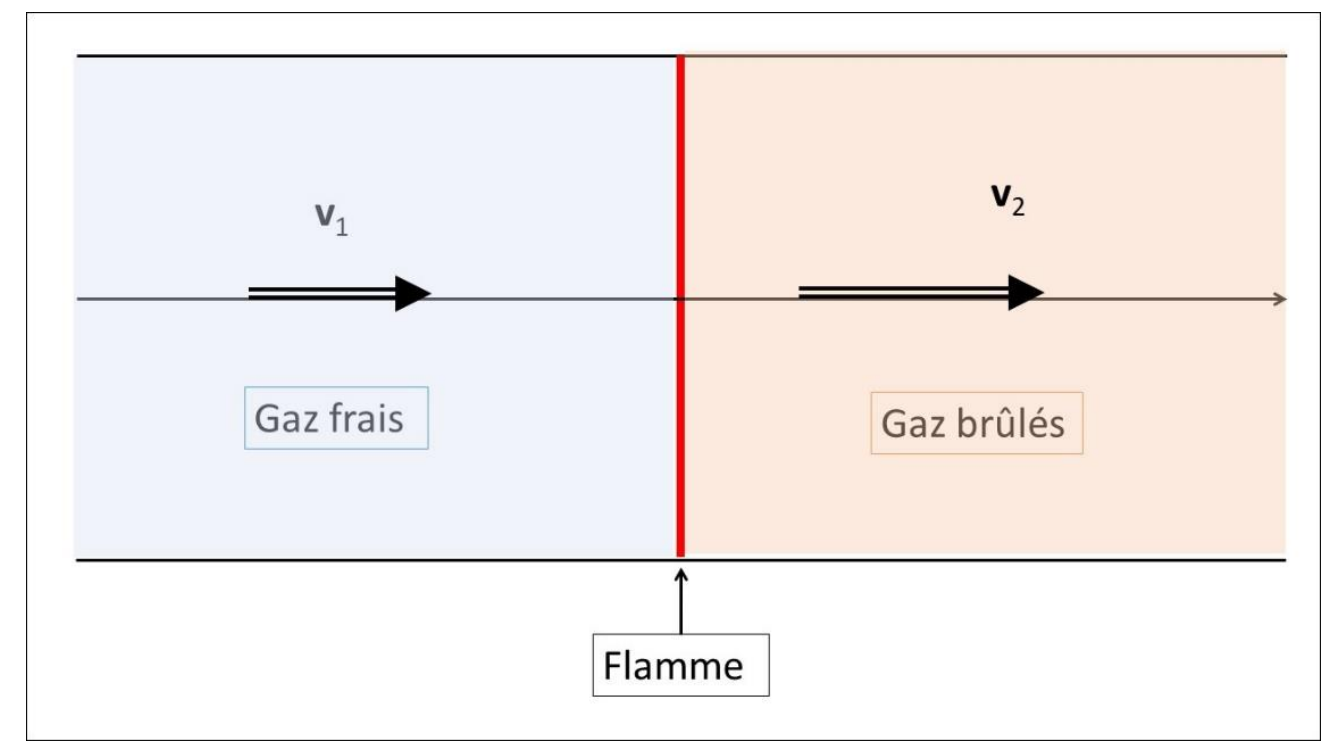

Figure 26. Configuration de Rankine-Hugoniot : représentation du mouvement stationnaire à l'échelle des dimensions de la tuyauterie dans un système lié à l'onde ; tuyauterie droite adiabatique remplie initialement d'un mélange de comburant et de combustible, $v_{1}$ est la vitesse de combustion laminaire adiabatique. Ici l'énergie d'activation de la réaction est très grande ce qui explique la minceur de la flamme.

Dans cette théorie simplifiée, les points solutions peuvent ainsi être obtenus graphiquement par l'intersection d'une courbe et d'une droite dans le plan volume-pression. La figure A7 25a montre les deux cas : la déflagration pour $M_{1}<1$ et la détonation pour $M_{1}>1$, le point de fonctionnement se trouvant à l'intersection de la droite de débit et de l'adiabatique d'Hugoniot qui est de forme hyperbolique.

L'onde de déflagration de vitesse $u_{1}=s_{L}{ }^{0}$ (L pour laminaire et ${ }^{0}$ pour adiabatique) correspond à un point de la branche $\mathrm{F}_{1} \mathrm{~F}$ où la variation de pression est faible et où l'augmentation du volume massique indique une forte dilatation. Mais trouver la position exacte de ce point n'est pas immédiat. C'est l'objet de la section 4.2 de l'article. 
Le point de détonation sur la branche $\mathrm{T}_{1} \mathrm{~T}_{2}$ est plus facile à déterminer. L'onde de détonation se produit au point $\mathrm{T}$, appelé point de Chapman-Jouguet de la détonation. On peut démontrer que ce point $\mathrm{T}$ où la droite de débit est tangente à la courbe d'Hugoniot est le seul stable de cette branche. Il suffit pour cela calculer analytiquement la variation d'entropie réduite $\left(s_{2}-s_{1}\right) / c_{\mathrm{v}}$ à la traversée de l'onde. Son évolution représentée sur la figure $7 \mathrm{~b}$ le long de l'adiabatique d'Hugoniot en fonction de la masse volumique réduite $\rho_{1} / \rho_{2}$ présente en effet un minimum en T. Nous ne présenterons pas plus ici l'onde de détonation dont sa vitesse est déterminée par les conditions au point $\mathrm{T}$. Les coordonnées du point $\mathrm{T}$ sont calculables analytiquement.

\section{Annexe 2}

\section{Les maîtres de la combustion}

Les écoulements avec réactions chimiques intéressent depuis longtemps les spécialistes de la combustion. Souvent les schémas théoriques étaient assez simples puisque l'on négligeait tantôt la convection comme dans le cas des déflagrations, tantôt au contraire l'aérodynamique était seule prise en compte comme dans les détonations, la chimie n'intervenant que par l'apport d'énergie de la réaction de combustion.

Dans bien des problèmes la situation est cependant devenue plus complexe et des disciplines aussi différentes que l'aérodynamique, la thermodynamique et la chimie interviennent simultanément et s'interpénètrent. C'est le cas avec la combustion dans les foyers industriels ou de propulsion, les problèmes de pollution, d'incendie, de pénétration des corps rentrant à très grande vitesse dans les atmosphères planétaires, ceux de couche limite réactive. On retrouve des situations semblables en métallurgie et dans certains réacteurs industriels.

Avec les travaux de recherche menés par plusieurs scientifiques est apparu le vocable d'Aérothermochimie, proposé par Théodore Von Karman, pour désigner cette nouvelle science. La combustion a accompagné l'évolution des connaissances qui s'est appuyée sur les travaux d'éminents précurseurs tels P. Duhem, F. Jouguet, Hirschfelder, I. Prigogine, S.R. De Groot, de L.G. Napolitano, Y.B. Zel'dovich. Des ouvrages importants ont été publiés. En combustion

on peut citer le livre de Williams ${ }^{\text {xviii }}$ puis ceux de Barrère ${ }^{\text {xxix }}$ et, avec un contenu actualisé sur les aspects turbulents, ceux de Peters (réf. ii) et de Poinsot (réf. iv).

L'Institut de la Combustion (en anglais : The Combustion Institute) est une société savante à but non lucratif, fondée en 1954 par Bernard Lewis et Hoyt Hottel, destinée à promouvoir les études dans le domaine de la combustion. Le Combustion Institute est présidé actuellement par James F. Driscoll. Il est basé à Pittsburgh aux États-Unis, et possède une trentaine de sections dans divers pays. La France est représentée par le Groupement Français de Combustion. L'institut organise un symposium annuel dont les travaux sont publiés et au cours duquel il distribue diverses récompenses.

Plusieurs revues sont en relation avec le Combustion Institute: Combustion and Flame, Proceedings of the Combustion Institute, Progress in Energy and Combustion Science, Combustion Science and Technology, Combustion Theory and Modelling.

Les photographies de trois scientifiques disparus, qui ont apporté beaucoup à la combustion, apparaissent sur la figure. 


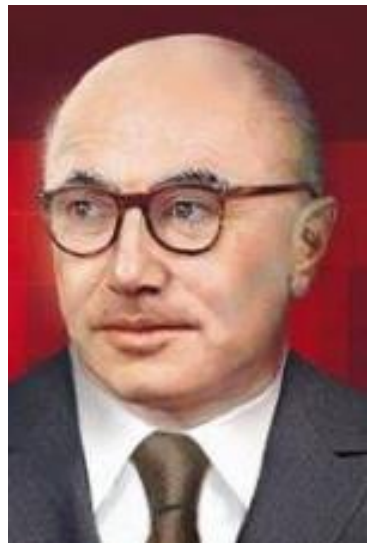

Yakov Borisovich Zel'dovich (1914-1987)

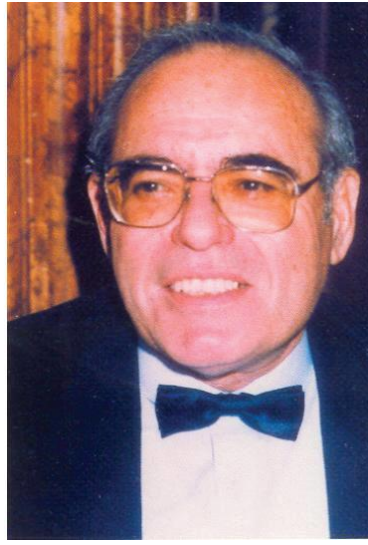

Marcel Barrère

(1920 - 1996)

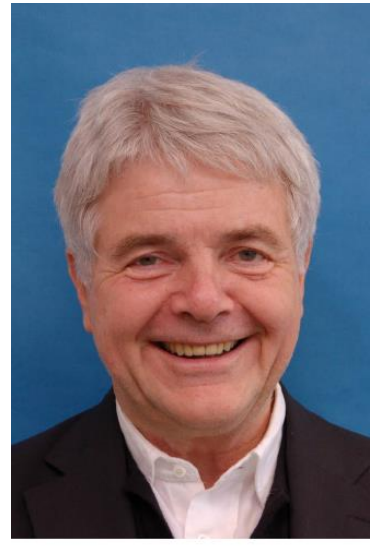

Norbert Peters $(1942-2015)$

De gauche à droite : lakov Borissovitch Zel'dovitch [Source : public domain ${ }^{18}$ ], Marcel Barrère [Source : Roger Prud'homme via Paul Kuentzmann], Norbert Peters [Source : public domain ${ }^{19}$ ]

- I.B. Zel'dovitch ${ }^{20}$ est un astrophysicien et physicien théoricien soviétique. Il développa la théorie des réactions en chaîne. Il fit d'importantes contributions dans les domaines de l'adsorption et de la catalyse, des ondes de choc, de la physique nucléaire, de la physique des particules, de l'astrophysique, de la cosmologie et de la relativité.

- M. Barrère est chercheur. Il fut Directeur scientifique de l'Energétique à l'Office National d'Etudes et de Recherches Aérospatiales, ONERA. Il a publié plusieurs ouvrages dont Rocket Propulsion ${ }^{\mathrm{xxx}}$ et Équations fondamentales de l'Aérothermochimie (réf. xxix).

- N. Peters était professeur à la RWTH Aachen University en Allemagne et l'une des autorités mondiales dans le domaine de l'ingénierie de la combustion. Il a dirigé l'Institut für Technische Verbrennung. Il a publié le livre de référence Turbulent combustion (réf. ii)

Sans vouloir faire une liste exhaustive, on peut ajouter les noms suivants :

- B. Lewis (1899-1993), déjà cité à propos de la création de l'Institut de la Combustion, est un chimiste américain d'origine britannique est une figure majeure de la combustion ${ }^{21}$. Il fut aussi dès 1946 le chef de la division Explosives and Physical Sciences du United States Bureau of Mines à Pittsburgh.

- Adolphe Van Tiggelen (1914-1969), éditeur d'un ouvrage en deux tomes Oxydations et combustions $^{\mathrm{xxxi}}$. Chercheur belge et professeur à l' Université Catholique de Louvain, UCL. Il a apporté sa contribution à la compréhension du processus de flamme. En 1961, il a eu le Francqui Prize sur les Sciences Exactes.

\footnotetext{
${ }^{18}$ https://commons.wikimedia.org/wiki/File:RUSMARKA-1827.jpg g

${ }_{19} \mathrm{https://www.google.fr/search?ei=MueJW4LJD} \mathrm{KUlwSdyo-IBQ \& q=norbert+peters \& oq.}$

20 On lui doit en particulier le nombre de Zel'dovich $Z e=E_{a}\left(T_{b}-T_{u}\right) / R T_{b}^{2}$ qui représente l'énergie d'activation réduite. $E_{a}$ est l'énergie molaire d'activation de la réaction chimique, $T_{b}$ et $T_{u}$ les températures respectives des gaz brûlés et des gaz frais, $\mathrm{R}$ est la constante molaire des gaz parfaits. Voir l'ouvrage Selected Works of Yakov Borisovich Zeldovich, Volume I Chemical Physics and Hydrodynamics, Princeton University Press, 1992. https://www.jstor.org/stable/j.ctt7zvs4j

21 On utilise couramment le nombre de Lewis pour comparer les effets de la conduction thermique à ceux de la diffusion des espèces chimiques : Le=k/D. 
- Luigi Gerardo Napolitano (1928-1991), directeur de l'institut aérodynamique de l'Université de Naples "Federico II", a enseigné aussi à Berkeley, à la Sorbonne et à Poitiers. Son champ de recherche particulièrement large comprenait la modélisation thermodynamique des mélanges gazeux multiréactifs et l'effet Marangoni en micropesanteur. Il fut président de la Fédération Internationale d'Astronautique. Il fut aussi le fondateur et le premier président du Microgravity Advanced Research and Support, MARS et du Centre italien des recherches aérospatiales, CIRA.

Il ne sera pas fourni ici de liste actualisée des combustionnistes d'aujourd'hui. Le lecteur pourra les trouver dans les entêtes des nombreux articles consultables dans les revues, citées ou non dans cette annexe. Il s'agit d'ailleurs souvent de travaux d'équipes plus que de travaux individuels. Quelques

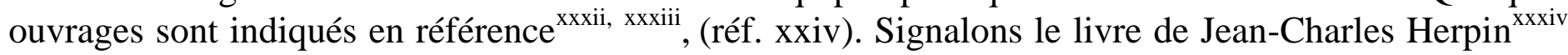
datant d'il y a deux siècles, qu'il peut être distrayant et instructif de feuilleter pour se faire une idée de l'expérimentation chimique à cette époque. Bien que n'étant pas un spécialiste de la combustion, J-C. Herpin a sélectionné des curiosités expérimentales dont certaines sont liées à la combustion.

\section{Références}

${ }^{\text {i }}$ Burke, S.P., Schumann, T.E.W. : Diffusion flames. In: First Symposium on Combustion, Swampscott, MA, 10-14 Sept. 1928.

ii Peters N., Turbulent combustion, (Cambridge Monographs on Mechanics), Cambridge University Press, Cambridge, 2000.

iii Williams, F.A.: Turbulent combustion, In: Buckmaster, J. (ed) The mathematics of combustion, SIAM, Philadelphia, 1985, pp. 97-131.

iv D., Poinsot T., Veynante Theoretical and numerical combustion, Second Edition, Edwards, 2005. ISBN 1930217056, 9781930217058

' Prud'homme R. (2015) Vaporisation et combustion de gouttes dans les moteurs, Editions Techniques de l'Ingénieur, Traité de Mécanique, BM 2 521v2, 29 p

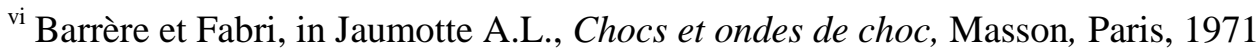

${ }^{\text {vii }}$ Prud'homme R., Flows of Reactive Fluids, Springer, 2010. ISBN 978-0-8176-4518-2.

viii Damköhler G., «Der Eifluss der Turbulenz auf die Flammengeschwindigkeit in Gasgemischen », Zeischrift für Elektrochemie, vol. 46, p. 601-652, 1940.

${ }^{\text {ix }}$ D. Hebert, A. Coppale, M. Talbaut, 2D soot concentration and burning rate of a vertical PMMA slab using LaserInduced Incandescence, Proceedings of the combustion institute 34 (2013) 2575-2582.

${ }^{\mathrm{x}}$ Emmons H.W., The film combustion of liquid fuel, Zeitschrift für angewandte Mathematik und Mechanik (ZAMM), vol 36, p. 60-71, 1956.

${ }^{\text {xi }}$ Dorey L.-H. « Modélisation des phénomènes couplés combustion - formation des suies - transferts radiatifs dans les chambres de combustion de turbine à gaz », Thèse ECP-ONERA, Paris, 01-06-2012.

xii J. Glorian, Cinétique hétérogène pour la combustion de l'aluminium, thèse de Doctorat de l'ENSTA_ParisTech, déc. 2014. HAL Id https://pastel.archives-ouvertes.fr/tel-01144079 Submitted on 20 Apr 2015.

${ }^{\text {xiii }}$ A.I. Kirdyashkin, V.D. Kitler, V. Salamatov, Y. M. Maksimov, Capillary hydrodynamic phenomena in gas-free combustion, January 2007. Combustion Explosion and Shock Waves 43(6) : 645-653. ISSN 1573-8345

${ }^{\text {xiv }}$ K.L. Klimenok, S.A. Rashkovskiy, Discrete model of gas-free spin combustion of powder mixture. Physical Review E Vol. 91(1), 012805, 2015. DOI: https://doi.org/10.1103/PhysRevE.91.012805

${ }^{\mathrm{xv}}$ K.L. Klimenok, S.A. Rashkovskiy, Discrete model of gas-free spin combustion of powder mixture. Physical Review E Vol. 91(1), 012805, 2015. DOI: https://doi.org/10.1103/PhysRevE.91.012805

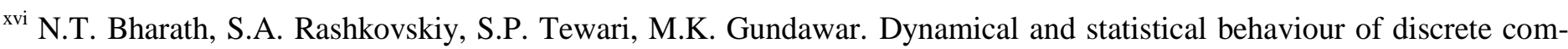
bustion waves: a theoretical and numerical study. Physical Review E Vol. 87, 042804, 2013. DOI : https://doi.org/10.1103/PhysRevE.87.042804 
${ }^{x v i i}$ C. Inoue, Y. Izato, A. Miyake, and E. Villermaux, Direct Self-Sustained Fragmentation Cascade of Reactive Droplets, Phys. Rev. Lett. 118, 074502 (2017).

${ }^{\text {xviii }}$ Yu. M. Maksimov, A. T. Pak, G. B. Lavenchuk, Yu. S.Malborodenko, A. G. Merzhanov, Spin combustion of gasless systems. Tomsk. Translated from Fizika Gorentya I Vzryva, Vol. 15, N 4, pp. 156-159, May-June 1979. Original article submitted June 28, 1978.

xix A.P. Aldushin, B.A. Malomed, Ya. B. Zeldovich, Phenomenological theory of spin combustion. Combustion and Flame 42: 1-6, 1981. https://doi.org/10.1016/0010-2180(81)90137-1

${ }^{x x}$ S.A. Rashkovskiy, G. Manoj Kumar and Surya P. Tewari, One-dimensional discrete combustion waves in periodical and random systems. Combust. Sci. and Tech., 182: 1009-1028, 2010. DOI: 10.1080/00102200903544263

${ }^{x x i}$ Combustion Explosion and Shock Waves. Publisher: Springer Verlag.

https://www.researchgate.net/journal/1573-8345_Combustion_Explosion_and_Shock_Waves

${ }^{x x i i}$ Modest M.F., Radiative heat transfer. Academic Press, 2nd edition, 2003.

xxiii Tessé L., Lamet J.M., "Radiative Transfer Modeling Developed at Onera for Numerical Simulations of Reactive Flows", Journal Aerospace Lab, Issue 2, p. 1-19, 2011.

${ }^{\text {xxiv }}$ Pollard A., Candel S. (eds.) IUTAM Symposium on Turbulent Mixing and Combustion, Kluwer Academic Publishers, Dordrecht (2002). ISBN 978-94-017-1998-8

${ }^{x x v}$ Jaumotte, A. L., Chocs et ondes de choc. Masson, 1971.

${ }^{x x v i}$ A. Lallemand, Techniques de l'Ingénieur, Sept. 2014, Réf : BE8165 v1

xxvii Barrère et Fabri, in Jaumotte A.L., Chocs et ondes de choc, Masson, Paris, 1971

xxviii Williams, F.A.: Combustion theory, 1st edn., Addison Wesley Publ. Cie, 1965, 2nd edn., Benjamin Cummings, Menlo Park, 1985. ISBN : 0-8053-9801-5

xxix Barrère M., Prud'homme R., Equations Fondamentales de l'Aérothermochimie, Masson et Cie, 1973. ISBN 978-2225-36003-9.

xxx Barrère, M. : La propulsion par fusées, Dunod, 1957. https://doi.org/10.1017/S0368393100069248 Published online : 01 July 2016.

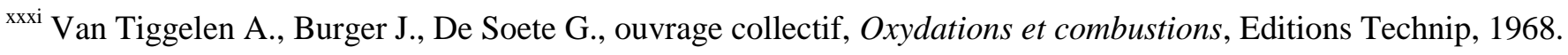

${ }^{x x x i i}$ Borghi R. , Champion M. Modélisation et théorie des flammes, Editions Technip, 2000. ISBN : 9782710807582

xxxiii Borghi R., Clavin P., Liñán A., Pelcé P., Shivashinsky G.I., ouvrage collectif, Modélisation des phénomènes de combustion. Eyrolles, 1985.

${ }^{\text {xxxiv }}$ Herpin J.-C., Récréations chimiques ou recueil d'expériences curieuses et instructives auxquelles on a joint un précis élémentaire de chimie, Audot, 1824. https://books.google.fr/books?id=E0F7zCAKzCoC . 